\title{
Evaluating the Calorimeter Model with Broadband, Continuous Spectra of Starburst Galaxies Observed with the Allen Telescope Array
}

\author{
Peter K. G. Williams \\ University of California, Berkeley, Department of Astronomy \\ 601 Campbell Hall \# 3411, Berkeley, CA 94720-3411 \\ pwilliams@astro.berkeley .edu \\ and \\ Geoffrey C. Bower \\ University of California, Berkeley, Department of Astronomy \\ 601 Campbell Hall \# 3411, Berkeley, CA 94720-3411 \\ gbower@astro.berkeley.edu
}

\begin{abstract}
Although the relationship between the far-infrared and $\mathrm{cm}$-wave radio luminosities of normal galaxies is one of the most striking correlations in astronomy, a solid understanding of its physical basis is lacking. In one interpretation, the "calorimeter model," rapid synchrotron cooling of cosmic ray electrons is essential in reproducing the observed linear relationship. Observed radio spectra, however, are shallower than what is expected of cooled synchrotron emission. In Thompson et al. (2006), a simple parameterized model is presented to explain how relatively shallow observed spectra might arise even in the presence of rapid synchrotron cooling by accounting for ionization losses and other cooling mechanisms. During the commissioning of the 42-element Allen Telescope Array, we observed the starburst galaxies M82, NGC 253, and Arp 220 at frequencies ranging from 1 to $7 \mathrm{GHz}$, obtaining unprecedented broadband continuous radio spectra of these sources. We combine our observations with high-frequency data from the literature to separate the spectra into thermal and nonthermal components. The nonthermal components all steepen in the cm-wave regime and cannot be well-modeled as simple power laws. The model of Thompson et al. is consistent with our M82 results when plausible parameters are chosen, and our results in fact significantly shrink the space of allowed model parameters. The model is only marginally consistent with our NGC 253 data. Assuming the Thompson et al. model, a steep electron energy injection index of $p=-2.5$ is ruled out in M82 and NGC 253 to $>99 \%$ confidence. We describe in detail the observing procedures, calibration methods, analysis, and consistency checks used for broadband spectral observations with the Allen Telescope Array.
\end{abstract}

\section{Introduction}

The correlation between the far-infrared (FIR) and centimeter-wave radio emission of normal galaxies has been recognized for almost four decades (van der Kruit 1971). It holds over more than four orders of magnitude in $L_{\mathrm{FIR}}$ (Yun et al. 2001), is constant out to at least $z=1$
(Appleton et al. 2004), and is observed in a large variety of galaxies (Condon 1992). The nearlinearity (Yun et al. 2001) and universality of the FIR-radio correlation strongly suggest that it is intimately linked to the fundamental astrophysics of galaxies.

The physical underpinning of the correlation is broadly understood to be the formation of 
massive stars, which can believably be associated with the dominant contributions to both the FIR and continuum cm-wave radio emission of galaxies Harwit \& Pacini 1975; Wunderlich \& Klein 1988). The FIR contribution is due to the hot dust envelopes surrounding these stars, which are energized by the copious amounts of Lyman continuum radiation that massive stars emit. The radio contribution includes two components. The first is synchrotron emission from relativistic cosmic ray (CR) electrons spiraling in the interstellar magnetic field. Such electrons can be accelerated in the supernova (SN) shocks that occur when massive stars die. The second, less significant, component is free-free emission, which predominantly comes from H II regions. These too are associated with massive stars.

This simple picture, however, neglects many important details. The timescales and dynamics of the radio and FIR emission are very different: CR electrons radiate over a timescale of $10^{4}-10^{7} \mathrm{yr}$ (see below), during which they diffuse through the galaxy, are possibly advected out of it via a galactic wind (Breitschwerdt et al. 1991), and cool via a variety of mechanisms, including inverse Compton (IC) scattering, ionization, free-free emission, and adiabatic expansion. Meanwhile, the FIR emission is prompt and comprised of comparable warm $(\sim 40 \mathrm{~K})$ and cool ("cirrus," $\sim 20 \mathrm{~K})$ dust components, the latter of which is primarily heated by the interstellar radiation field, not newly-formed stars (Walterbos \& Schwering 1987; Boulanger \& Pérault 1988; Fitt et al. 1988).

Much theoretical and observational effort has been expended in the cause of understanding the FIR-radio correlation in detail, (e.g. de Jong et al. (1985); Helou et al. (1985); Cox et al. (1988); Hummel et al. (1988); Devereux \& Eales (1989); Price \& Duric (1992); Lisenfeld et al. (1996)). An influential model has been that of Völk (1989), which presents a theory for understanding the FIR-radio correlation in the case that CR electrons are accelerated only by $\mathrm{SNe}$ and cool completely within a galaxy. In this situation, the galaxy acts as a "calorimeter" with regard to the energy injected into CR electrons. For the FIR-radio correlation to hold, the synchrotron luminosity per unit energy injected into CR electrons must be a universal constant. Taking the primary cooling mechanisms to be IC scattering and synchrotron, it is found that $\bar{U}_{B} /\left(\bar{U}_{\mathrm{ph}}+\bar{U}_{B}\right)$ must be nearly a constant value, where $\bar{U}_{B}=\left\langle B^{2} / 8 \pi\right\rangle$ is the mean energy density of the interstellar magnetic field and $\bar{U}_{\mathrm{ph}}$ is the mean energy density of the interstellar radiation field.

Because the synchrotron cooling time scales as $B^{-3 / 2}$ (Thompson et al. 2006, hereafter T06), the applicability of the calorimeter model depends on the strength of the interstellar magnetic field. In a stronger field, the calorimeter model is more likely to hold as CR electrons will cool more rapidly and have less chance to escape the galactic disk before radiating all of their endowed energy. This is precisely the scenario suggested in $\mathrm{T}+06$, where it is argued that starforming galaxies have much stronger magnetic fields than commonly assumed. The work assesses the applicability of the calorimeter model by considering the timescales for synchrotron cooling and escape from the galactic disk by CR electrons. Using the usual assumptions, the two are comparable; using the stronger magnetic field that $\mathrm{T}+06$ argues for, the synchrotron cooling timescale is much shorter, favoring the assumptions of the calorimeter model.

$\mathrm{T}+06$ specifically argues that in starburst galaxies the magnetic field is dynamically relevant: its energy density is comparable to the hydrostatic pressure of the ISM, or

$$
B \approx B_{\mathrm{eq}}=\left(8 \pi^{2} G\right)^{1 / 2} \Sigma_{g} \approx 2.3\left(\frac{\Sigma_{g}}{1 \mathrm{~g} \mathrm{~cm}^{-2}}\right) \mathrm{mG},
$$

where $\Sigma_{g}$ is the surface density of the galactic gas disk. This is in contrast to the classic "minimumenergy" estimate (Burbidge 1956; Longair 1994),

$$
B \approx B_{\min } \approx 9.5 \eta^{2 / 7}\left(\frac{L_{\nu}}{10^{23} \mathrm{~W} \mathrm{~Hz}^{-1}}\right)^{2 / 7}\left(\frac{V}{1 \mathrm{pc}^{3}}\right)^{-2 / 7}\left(\frac{\nu}{1 \mathrm{GHz}}\right)^{1 / 7} \mathrm{r}
$$

where $\eta$ is the ratio of total CR energy to energy in the $\mathrm{CR}$ electrons alone, $L_{\nu}$ is the synchrotron luminosity, $V$ is the estimated volume of the emitting region, and $\nu$ is the observing frequency. For the parameter values commonly associated with starburst galaxies, $B_{\text {eq }} \gg B_{\min }(\mathrm{T}+06$, fig. 1$)$.

An important observational constraint on the argument of $\mathrm{T}+06$ is the shape of the galactic nonthermal spectrum. It is typically argued that galactic spectra are insufficiently steep to be compatible with the calorimeter model: observations indicate $\alpha \approx-0.8\left(S_{\nu} \propto \nu^{\alpha}\right.$; Condon (1992); 
Niklas et al. (1997)) rather than $\alpha \approx-1$ as would be expected from the combination of SN shock physics (Blandford \& Eichler 1987) and spectral steepening caused by strong cooling (Condon 1992). T+06 contends that more detailed analysis mitigates this issue. As CR electron energy decreases, the timescales of ionization losses and free-free emission are, respectively, shorter and unchanged, so both of these processes will tend to flatten observed spectra. In $\mathrm{T}+06$ simple steadystate simulations were performed to quantify this effect (cf. its fig. 3 and $\S 4.3$ ). These simulations lead to the general prediction that the nonthermal radio spectra of starburst galaxies should steepen with increasing frequency, asymptoting to $\alpha \lesssim-1$.

Most spectral index measurements are based on only a handful of fluxes. To accurately measure the steepening of a nonthermal spectrum, many more data are needed, because both the steepening and the thermal contribution must be determined. The work of Duric et al. (1988) and Niklas et al. (1997) shows that determination of the thermal fraction is difficult and requires high-quality highfrequency data, even if a simple power-law nonthermal spectrum is assumed. As Condon (1992) points out, the thermal fraction and nonthermal steepening can also be highly degenerate (cf. his Figure 6). One must sample a large number of points over a wide frequency range in uniform conditions to effectively constrain these parameters.

Though it is still being commissioned, the Allen Telescope Array (ATA; Welch et al. (2009)), with its continuous frequency coverage of $0.5 \mathrm{GHz}$ to $10 \mathrm{GHz}$, is well-suited to testing the predictions of $\mathrm{T}+06$ by observing high-resolution, continuous spectra of starburst galaxies. Section 2 describes observations of M82, NGC 253, and Arp 220 made with the ATA in the fall and winter of 2008. In $₫ 3$ our data reduction methods are described along with results for standard calibrator sources. Section 4 describes our modeling of the observed spectra to extract thermal and nonthermal contributions. Finally, in $\$ 5$ we discuss our results and in \$6] we present our conclusions.

\section{Observations}

We observed the starforming galaxies M82, NGC 253, and Arp 220 with the Allen Telescope Array on seven nights over the course of 2008
September to 2009 June. The ATA is a large- $N$, small- $D$ (LNSD) cm-wave array located in Hat Creek, California, and is a joint project of the SET 1 Institute of Mountain View, CA and the Radio Astronomy Laboratory of the University of California, Berkeley. The scientific motivators and technical capabilities of the ATA are described in Welch et al. (2009). At the time of the observations the ATA was undergoing commissioning, with many aspects of the array being actively developed. Forty-two antennas and one correlator had been installed at the site. Two-thirds of the antennas were typically available for use during our observations.

We used the continuous frequency coverage of the ATA to observe the galaxies at almost a decade of sky frequency in a sequence of snapshot observations. The observing frequencies ranged between $1.0 \mathrm{GHz}$ and $7.0 \mathrm{GHz}$ with a spacing of $100 \mathrm{MHz}$, skipping some frequencies due to known radiofrequency interference (RFI) or low priority. The primary beam sizes of the ATA at $1.0 \mathrm{GHz}$ and $7.0 \mathrm{GHz}$ are $\sim 210^{\prime}$ and $\sim 30^{\prime}$, respectively, and the synthesized beam sizes are typically $\sim 250^{\prime \prime}$ and $\sim 35^{\prime \prime}$. The snapshots lasted between 60 and 130 seconds, with the correlator dump time always being 10 seconds. All observations used a bandwidth of $104.9 \mathrm{MHz}$ and 1024 correlator channels and sampled both vertical and horizontal linear polarizations. Observations of the science targets were interleaved with observations of the calibrators 3C 48, 3C 147, and 3C 286. These calibrators are compact to the ATA and have up-to-date broadband flux density models available from the VLA 2 Because of the need to set the flux density scale at numerous frequencies and the desire to obtain a large amount of data for sanity-checking, a large portion of the observing time was dedicated to calibrator observations. The total integration time on the calibrators was ten hours, compared to nine hours spent on the target galaxies. An observing strategy that gathered fewer redundant data could spend approximately half as much time observing calibrators as observing science targets.

${ }^{1}$ Search for Extraterrestrial Intelligence ${ }^{2}$ http://purl.oclc.org/net/pkgwpub/vla-analytic-cal-models 


\subsection{Details of observing methods}

Because the observations presented herein use a novel technique on a novel instrument, we shall describe our methods in detail. Each night was divided into several segments during which a subset of the targets and calibrators would be observed as a group. The typical duration of a segment was a few hours. Over the course of each segment, an observing script would repeatedly slew to each of the selected sources in turn, observing each at several frequencies before moving on to the next, until the segment's allotted time had elapsed. This scheme attempted to balance the desire to obtain calibrator observations temporally close to the science observations with the desire to not spend too much time moving from one target to the next. Most segments included observations at $1.4 \mathrm{GHz}$ and $5.0 \mathrm{GHz}$ to enable checks of repeatability and stability. The observations presented in this paper are summarized in Table 1 .

Observing over a large range of frequencies requires not just a large number of calibrator scans but also reconfiguration of the ATA hardware. In particular, the sensitivity of the log-periodic ATA feeds is a function of position along the axis of their pyramidal structure, with the point of peak sensitivity approaching the apex of the pyramid with increasing frequency. To allow for maximal performance, the ATA feeds can be focused by moving them in a piston motion along the normal to each antenna's focal plane (see Welch et al. 2009, fig. 4). The optimal focus positions have been determined theoretically and calibrated so that an ATA observer can specify the desired focus position in frequency space. The focusing operation, however, is slow. In our work, before each observation we set the feed focus frequency to the observing frequency rounded up to an integral number of $\mathrm{GHz}$ (e.g., 4.0, 4.4, and $4.9 \mathrm{GHz}$ all map to 5 $\mathrm{GHz}$ ). This scheme was chosen because the sensitivity of the feed to frequencies above the nominal focus frequency drops off more rapidly than the sensitivity to frequencies below it. Within each segment we chose observing frequencies that lay within a single $\mathrm{GHz}$ span to avoid superfluous refocus operations. The focusing and other timeconsuming operations are parallelized as much as possible to conserve observing time.

Because neither the feed sensitivity nor the noise power entering the ATA are spectrally flat, when the focus position of a feed changes, the total power entering the analog system also changes. Just before digitization, the downmixed and filtered analog signal is passed through an "attemplifier" (attenuator/amplifier) stage to adjust the incoming power for optimal quantization (Backer 2007). The amount of attenuation or amplification must be explicitly configured, but the best settings can be calibrated automatically during observations. Clearly, any two observations that are to be compared or used for flux calibration must be made with the same attemplifier settings. Furthermore, the attemplifier calibration step is also somewhat slow $(\sim 60 \mathrm{~s})$. In these observations, snapshots made at similar frequencies shared attemplifier settings. Specifically, one set of attemplifier settings was used for each integral $\mathrm{GHz}$ span (e.g., $4 \leq \nu_{\mathrm{GHz}}<5$ ). The first time any frequency in a span is observed, the attemplifier settings are calibrated for that frequency and saved; for subsequent observations during the same segment, the saved settings are reapplied.

As an example of all of these considerations, consider segment \#12, as detailed in Table2. This segment was scheduled for observation from 03:42 UT to 05:12 on 2008 Oct 15 . (All dates and times are in UT.) The selected sources were $3 \mathrm{C} 48$, 3C 147, and M82. The timing and (short) duration of this segment were set by the visibility of the science targets to the ATA: Arp 220 set just before this segment began, and NGC 253 rose just after it ended. Because M82 is circumpolar at the ATA's latitude, the other two targets were prioritized whenever they were visible. The selected observing frequencies were 1.4, 2.4, 2.5, 4.3, 4.7, and $5.0 \mathrm{GHz}$. As described above, the observing script slewed to the sources in turn and observed each at several frequencies. The frequencies were chosen and grouped so as to reduce the amount of time spent adjusting the feed focus positions and attemplifier settings. At 04:08, the script would have observed 3C 147 at the trio of lower frequencies if possible, but that source had not yet risen. Instead, the script skipped those observations and looped back to observe 3C 48 at the trio of higher frequencies.

Including time lost due to slewing, hardware initialization, software overhead, and so on, we made $\sim 18$ snapshots per hour while observing, in- 
tegrating for $38 \%$ of the 53 hours spent using telescope resources during the observations presented in this paper. The efficiency of future observations will be higher: we have made an unusually large number of calibrator observations and have made no effort to reduce slew time between sources. Furthermore, our observations were made before the installation of a second independently-tunable correlator at Hat Creek, which doubles the observational throughput.

\section{Data Reduction}

The data were reduced with a combination of the analysis package MIRIAD 3 (Sault et al. 1995) and custom Python 4 scripts based on the NumPy and IPython packages. Over the course of this project and other work on the ATA, significant improvements have been made to MIRIAD to ease its compilation, improve its ability to handle large datasets, and fix various bugs. A special module was written to allow easy management of and access to MIRIAD datasets in the Python language 5

The two orthogonally-polarized signals that are received by the ATA feed elements are amplified by separate low-noise amplifiers (LNAs) and subsequently treated as entirely separate signals. The ATA correlator at the time of the observations accepted 64 such antenna-polarization signals, or "antpols" for short, making it capable of correlating 32 antennas with full-polarization coverage, 64 antennas with single-polarization coverage, or, as encountered in practice, an intermediate case. Each ATA antenna is identified by a small integer and a single letter, e.g. $3 \mathrm{~g}$. (These specify the node and element identifier of the antenna within the ATA architecture.) An antpol is specified by suffixing the antenna name with the letter $\mathrm{X}$ or $\mathrm{Y}$, e.g. $2 \mathrm{kY}$. We refer to a pair of antpols as a "basepol."

Although the ATA correlator produces crosshand polarization data, the calibration and analysis of these data have not yet been investigated and tested at the time of this work. In this paper, cross-hand data were discarded, and all sources were assumed to be completely unpolarized. All

$3^{3}$ http://purl.oclc.org/net/pkgwpub/miriad

${ }^{4}$ http://www.python.org/

${ }^{5}$ http://purl.oclc.org/net/pkgwpub/miriad-python images produced were of the Stokes I parameter. Because the linear polarization of $3 \mathrm{C} 286$ is $\sim 10 \%$, this treatment will lead to $\sim 1 \%$ errors in some measurements. This effect contributes to, but does not dominate, the typical uncertainty in our measurements (see \$3.4).

\section{1. $\quad$ Flagging}

The ATA does not yet have an online flagging system and our observations were performed outside of the protected bands. Fairly extensive manual editing of the data was performed. Several automatic criteria were applied. The 100 channels on both ends of the 1024-channel spectral window were flagged because the ATA digital filter aliases in large amounts of noise at the bandpass edges. Shadowed antennas were flagged, taking the effective antenna radius to be $7.5 \mathrm{~m}$ (somewhat more than the $6.1 \mathrm{~m}$ physical diameter of the primaries). Finally, visibilities with $\sqrt{u^{2}+v^{2}}<100 \lambda$ were flagged as these short baselines were consistently sensitive to terrestrial and solar RFI, undesired large-scale structure, and possible cross-talk.

A fraction of the antpols had problems with their cryogenics, LNAs, or delay calibrations, resulting in data for the night that could easily be rejected after a cursory look. Occasional correlator hardware failures resulted in wildly erroneous amplitudes in a small subset of the spectral data, e.g. the first quarter of the spectrum of all visibilities involving antpols $1 \mathrm{aX}, 1 \mathrm{cY}$, and $4 \mathrm{kX}$. Such data were also flagged and the issue reported.

After an initial check for bad antpols or correlator hardware, RFI was flagged with a semiautomated, channel-based routine. All of the amplitude spectra at a given frequency observed over the course of a single segment were summed, regardless of time, baseline, polarization, or pointing. A simple peak-finding routine identified suspected RFI channels by searching for extremely high amplitudes. The suspect channels were presented to the user graphically and made available for interactive editing before a list of channels to flag was recorded. The listed channels for each frequency were flagged out for all observations at that frequency in the segment. Figure 1 shows an example of the summing and peaking-finding process. Figure 2 characterizes the RFI environment of Hat Creek via a schematic representation of the summed amplitudes across the entire observed fre- 
quency range.

This procedure is obviously suboptimal in the case of time-varying RFI, but it was found that most of the interference was virtually always present. Informal tests of more sensitive methodologies that attempted to better mitigate timevariable RFI failed to improve results and were much more time-intensive to the user.

A few bands had nearly omnipresent RFI that made astronomical observations nearly impossible. These were: $500-1000 \mathrm{MHz}$ (terrestrial television, radar, pagers, aeronautical navigation, selfinterference), portions of L band (1150-1280 MHz, aeronautical navigation and GPS L5; 1520-1610 $\mathrm{MHz}$, which includes GPS L1 at $1575 \mathrm{MHz}$ ), the lower end of $\mathrm{S}$ band (satellite communications, 2150-2350 MHz, especially Sirius XM radio centered at $2339 \mathrm{MHz}$ ), and $\mathrm{C}$ band (satellite downlinks, 3700-4200 MHz).

After RFI-afflicted channels were flagged, various manual data-quality checks were run to check for remaining issues. These included phase-closure rankings of the calibrator observations, evaluation of the amplitude and phase variability of the baselines, and by-eye assessments of spectra. Many of these checks resulted in a few antpols or basepols being flagged for the duration of the segment, while occasionally a temporary system glitch was revealed and edited out.

Finally, after system temperatures were computed (see below), antpols with a $T_{\text {sys }}$ above 300 $\mathrm{K}$ were flagged. At the end of the flagging process, $\sim 40 \%$ of the antpols and $\sim 75 \%$ of the visibility channels in a typical dataset were discarded. Figure 3 shows the average data retention rates as a function of frequency. The deleterious effect of RFI is apparent, as is the moderate degradation of system performance with increasing frequency. Approximately 30\% fewer antpols and visibilities are retained at $7 \mathrm{GHz}$ as compared to $1.4 \mathrm{GHz}$.

Ongoing retrofits to the array are increasing the amount of usable data generated during each observation. A large portion of this work comprises improved RFI mitigation techniques, including better shielding, especially of the signal processing room; improved techniques and algorithms for interference excision; and stricter onsite RFI protocols. Some data are lost because certain antpols have extremely poor system tem- peratures, for various reasons: failed cryogenics systems, damaged LNAs, or offset pointing due to imbalanced electrical connections. More robust replacement hardware will make these issues much rarer.

\subsection{Calibration}

The calibration of our data is challenging because of the modest $u-v$ coverage, short integration times, and wide temporal and spatial separation between snapshots entailed by our observing mode. We will provide an overview of our calibration process with more detailed descriptions presented in the subsections below.

The snapshot observations of the calibrator sources were first self-calibrated to point-source models. All observations of the same calibrator and frequency were then imaged jointly to form a self-calibrated reference image with $u-v$ coverage and sensitivity superior to those obtained in any one snapshot. The individual calibrator snapshots were then recalibrated to their corresponding reference images.

The snapshot observations of the science sources then had appropriate calibration solutions applied to them. Some snapshots (of both science targets and calibrators) were duplicated and had several different calibration solutions applied to allow for cross-checks of the calibrator flux models. Reference images were then generated from the calibrated observations and once again the individual snapshots were recalibrated to their corresponding reference images.

Given the nature of this work, accurate broadband calibrator flux density models are vital. The 1999.2 analytic models listed in the VLA Calibrator Manual were initially adopted and found to yield good results. These models were, however, almost a decade old at the time of this work, and long-term VLA monitoring indicates that the fluxes of the calibrators evolve slightly on this timescale. Section 3.6 describes consistency checks used to verify and fine-tune the flux density scale used in this work.

\subsubsection{Self-calibration}

The calibration process started with selfcalibration of the calibrator observations. Bandpasses and complex antenna gains in the indi- 
vidual snapshots were first determined via the MIRIAD task smamfcal, which assumes a pointsource model. Spectral smoothing was used to increase the signal-to-noise ratio (SNR) in the high-frequency observations.

System temperature $\left(T_{\text {sys }}\right)$ information was then computed and inserted into the datasets. This step was necessary because the ATA did not have online system temperature calibration at the time of this work. A Python script called calctsys was developed to estimate $T_{\text {sys }}$ values from the variances in the real and imaginary parts of the visibilities across the band. The variances were used to compute per-basepol $T_{\text {sys }}$ values assuming an antenna gain of $153 \mathrm{Jy} / \mathrm{K}$. Per-antpol $T_{\text {sys }}$ values were then computed by performing a least-squares minimization assuming that the $T_{\text {sys }}$ of each basepol was the geometric mean of the $T_{\text {sys }}$ of its two contributing antpols. The values obtained in this way had good reproducibility over the course of a segment and agreed well with lunar measurements made by ATA staff (R. Forster, private communication). The system temperatures could vary strongly as a function of frequency, with most, but not all, antpols demonstrating superior performance at lower frequencies.

Reference images were then created from the group of calibrator snapshot observations. All of the snapshots of each source and frequency were merged into a temporary dataset that was imaged using natural weighting. Multifrequency synthesis (Conway et al. 1990) was used to avoid bandwidth smearing and provide better $u-v$ sampling. The images were deconvolved with the MIRIAD implementation of the CLEAN algorithm Högbom 1974), which incorporates the algorithmic adjustments suggested by Clark (1980) and Steer et al. (1984). After an initial round of imaging, the merged visibilities were self-calibrated to the generated CLEAN component model and imaged once more. The second self-calibration was phase-only if the SNR of the data was less than 0.3. Testing showed that further iterations of calibration and imaging did not lead to improvements in the image quality. Figure 4 shows the reference image of NGC 253 at $1.3 \mathrm{GHz}$ and an image generated from a single snapshot observation of the same source at the same frequency. Figure 5 shows reference images of M82 at four frequencies. The individual calibrator snapshots were then recalibrated using the CLEAN component model of the reference images.

\subsubsection{Secondary calibration}

The complex antenna gains and bandpass solutions were copied from each calibrator snapshot to any observations made at the same frequency within 40 minutes of the calibrator observation, including observations of different calibrators. To allow further consistency checks, if multiple calibrator observations could be applied to a given snapshot, it was duplicated and a different calibration was applied to each copy. The copy with the smallest time differential between the two observations was treated as the "official" version of the observation, with the other copies not being included in the final analysis. The median time differential of the "official" observations was 21 minutes, with values ranging from 3 to 38 minutes. The stability of the ATA over such timescales was qualitatively very good. Such stability is particularly noteworthy because of the large angles on the sky separating the calibrator and target sources (see, e.g., Table 2).

Once the observations of the science targets were flux and bandpass calibrated, system temperatures were computed and inserted into the datasets using calctsys. If the science targets had bright, off-center emission, the calctsys technique would have computed incorrect $T_{\text {sys }}$ values due to significant visibility variations across the passband, but this was not an issue for our selected sources.

Reference images of the science targets were then generated from the calibrated observations in a manner similar to that described above. A reference image was generated for each observed combination of source, sky frequency, and calibrator. Before imaging, the merged data were phase self-calibrated against a primary-beam-corrected model generated from the NRAO VLA Sky Survey (NVSS; Condon et al. (1998)) catalog. The purpose of this step was to compensate for occasional antenna-based phase glitches that could occur between the calibrator observation and the observation it was applied to. To roughly correct for the fact that the NVSS data only comprise a single frequency, each NVSS model image was scaled by $S_{\nu}(\nu) / S_{\nu}(1.4 \mathrm{GHz})$ before the phase self-calibration, where $\nu$ was sky frequency 
of the ATA observations and $S_{\nu}(\nu)$ was a simplistic model of the spectrum of the source being observed. The NVSS catalog sources corresponding to NGC 253 was found to poorly reflect its extended structure, so a bootstrapped model of that source's spatial emission was used rather than the NVSS model. After this first round of phase selfcalibration, the $u-v$ data were imaged and recalibrated as described above.

\section{3. $u-v$ modeling}

Fluxes were determined by fitting the $u-v$ data with point-source and elliptical Gaussian models based on the reference images. Our measurements are listed in Tables 35 . The calibrators and Arp 220 were all well-modeled as point sources. M82 was modeled as an elliptical Gaussian. The deconvolved source sizes determined for M82 varied slightly over the range of frequencies observed. The major (minor) axis of M82 measured approximately 60 (40) arcsec at $1 \mathrm{GHz}$ and 35 (20) arcsec at $7 \mathrm{GHz}$, with a roughly constant position angle (PA) of $\sim 80^{\circ}$ (east from north). The higherfrequency values are consistent with observations made at 1.48, 4.87, 32, and $92 \mathrm{GHz}$ (Seaquist et al. 1985; Klein et al. 1988; Carlstrom \& Kronberg 1991). The lower-frequency values are likely biased by the large $\left(\sim 250^{\prime \prime}\right)$ synthesized beam of the ATA at low frequencies.

NGC 253 was modeled as the superposition of an elliptical Gaussian and a more compact source. At lower frequencies, the field of view contained bright sources away from the pointing center which were also modeled. The size of the extended emission is strongly frequency-dependent, having a major (minor) axis of 480 (200) arcsec at $1 \mathrm{GHz}$ and $200(50)$ arcsec at $7 \mathrm{GHz}$, with roughly constant PA of $\sim 50^{\circ}$. The morphology of this region at $1.5 \mathrm{GHz}$ is consistent with the "inner disk" identified in Hummel et al. (1984). One should bear in mind that the spectra presented in Table 4 and Figure 10 manifest not only the changing brightness of this region but its changing size as well. The more compact source was unresolved at lower frequencies and modeled as a point source. Above $3.5 \mathrm{GHz}$, however, it was possible for some structure in the compact source to be discerned, and it was found that an elliptical Gaussian with major (minor) axes of $\sim 16(\sim 10)$ arcsec at a PA of $\sim 60^{\circ}$ was a practical model. This morphology is consistent with the "nuclear peak" seen in the near IR by Scoville et al. (1985).

The residuals to the fitted $u-v$ models were imaged to check for missed sources, poorly calibrated baselines, and so on. Further flagging was usually sufficient to solve any issues. Some datasets with severe RFI were not recoverable and had to be discarded.

Figure 6 shows a histogram of the fractional flux residuals of the calibrator snapshots as compared to the analytic calibrator models. The calibrator fluxes are those derived from $u-v$ modeling of calibrator snapshots that have been self-calibrated to the calibrator reference images. The vast majority of the measurements agree to within 1\%, demonstrating that the reference-image calibration pipeline propagates fluxes with high fidelity.

\subsection{Uncertainties}

The MIRIAD $u-v$ fitting task, uvfit, reports statistical uncertainties, but these fail to account for systematics and are only lower limits. We augmented these uncertainties with both relative and absolute terms:

$$
\sigma_{i}^{2}=s_{i}^{2}+\left(\eta S_{\nu, i}\right)^{2}+\left(\sigma_{a} \cdot 1 \mathrm{Jy}\right)^{2},
$$

where $\sigma_{i}$ is the uncertainty used for the $i^{\text {th }}$ measurement, $s_{i}$ is the uncertainty for that measurement reported by the fitting routine, $S_{\nu, i}$ is the value of the measurement, and $\eta$ and $\sigma_{a}$ are tunable parameters.

The choice of these parameters was derived from the statistics of the ensemble of observations. For each observed combination of source, frequency, and calibrator, the weighted mean of the contributing observations was computed:

$$
\bar{S}_{\nu}=\sum_{i=1}^{n} S_{\nu, i} \sigma_{i}^{-2} / \sum_{i=1}^{n} \sigma_{i}^{-2} .
$$

Let there be $N$ observed combinations of source, frequency, and calibrator, with $n_{j}$ observations of the $j^{\text {th }}$ combination. Let $\bar{S}_{\nu, j}$ be the weighted mean of the $n_{j}$ observations, and $S_{\nu, j, i}$ and $\sigma_{j, i}$ the values and uncertainties of the individual observations. If the uncertainties have been correctly assessed and the measurements are drawn from normal distributions, the sum of the squares of 
the normalized residuals,

$$
K^{2}=\sum_{j=1}^{N} \sum_{i=1}^{n_{j}}\left(\frac{S_{\nu, j, i}-\bar{S}_{\nu, j}}{\sigma_{j, i}}\right)^{2}
$$

should be taken from a $\chi_{k}^{2}$ distribution, where $k=$ $\sum_{j=1}^{N} n_{j}-N$ is the number of degrees of freedom. The expectation value of such a distribution is $k$.

The value of $K^{2}$ was computed on a grid of $\eta$ and $\sigma_{a}$ for three groups of measurements: the full ensemble, those with $\bar{S}_{\nu}<1$ Jy ("faint"), and those with $\bar{S}_{\nu}>3$ Jy ("bright"). Figure 7 shows contours of $K^{2}=k$ (i.e., uncertainties agreeing with observed scatter) in the $\eta-\sigma_{a}$ plane. As expected, the contours of constant $K^{2}$ are primarily along the $\eta$ axis for the faint group (for which $\sigma_{a}$ dominates the additional uncertainty) and primarily along the $\sigma_{a}$ axis for the bright group (for which $\eta$ dominates the additional uncertainty). The $K^{2}=k$ contours for the three groups intersect at an approximate location of $\eta=0.030$ and $\sigma_{a}=0.014$, i.e. an additional $3.0 \%$ fractional uncertainty and $14 \mathrm{mJy}$ absolute uncertainty. These are the values of $\eta$ and $\sigma_{a}$ that were adopted. The value of $\sigma_{a}$ is approximately equal to the thermal noise in good conditions and is about half of the thermal noise at high frequencies, where $T_{\text {sys }}$ generally increases.

If the uncertainties are correctly assessed and measurements are drawn from normal distributions, the sum of the normalized residuals of each measurement is drawn from a normal distribution with mean 0 and variance $\sigma^{2}=\sum_{j=1}^{N} n_{j}$. For the chosen parameter values, the sum of the residuals was $0.52 \sigma$. This moderate value suggests that the adopted uncertainties characterize well the observed scatter in the measurements and that the weighted-mean approach does not mask large variations in the individual measurements.

\subsection{Spectra}

We constructed broadband, high-resolution spectra out of the flux measurements described in 3.3 Repeated measurements were combined by taking a weighted mean as described in the previous section. Twenty-five percent of the spectral data are based on a single observation, while $63 \%$ are based on at least three observations. The maximal number of contributing observations is
13, of M82 at 5.0 GHz. The uncertainty of each spectral point is the propagated uncertainty of the weighted mean,

$$
\sigma_{S, j}^{2}=\left(\sum_{i=1}^{n_{j}} \sigma_{j, i}^{-2}\right)^{-1}
$$

The spectra are shown in Figures 8, 11] along with measurements from the literature and model results as discussed in 94 .

\subsection{Self-consistency Checks}

We performed no absolute flux calibration of the ATA. The measurements we have made are thus measurements of flux density ratios, with absolute flux densities being derived from a priori spectral models of the calibrator sources. We cannot verify the models themselves, but we can assess whether our measured flux ratios are consistent with them.

Let the "true" flux density of source $X$ (i.e., the value that would be measured by a perfect, absolutely calibrated antenna at the time of observation) be $T_{X}$, the a priori modeled flux density of $X$ be $M_{X}$, and the flux density of $X$ observed by the ATA after calibration to source $Y$ be $O_{X, Y}$. All of these values vary with frequency. In the simplest possible model, at a fixed frequency $O_{X, Y}$ and $T_{X}$ are directly related by a calibrator-dependent gain factor $g_{Y}$ :

$$
O_{X, Y}=g_{Y} T_{X}
$$

The gain is self-calibrated such that $O_{Y, Y} \equiv M_{Y}$, so that ideally

$$
O_{X, Y}=M_{Y} \frac{T_{X}}{T_{Y}} \equiv M_{Y} R_{X, Y}^{T},
$$

where $R_{X, Y}^{T}$ is the true flux density ratio of $X$ to $Y$. Not knowing whether this simple model is correct, we will define the observed flux ratio as

$$
R_{X, Y}^{O} \equiv \frac{O_{X, Y}}{M_{Y}} .
$$

If this simple calibration model holds, the observed ratios should demonstrate consistency and closure:

$$
\begin{gathered}
R_{X, Y}^{O} \cdot R_{Y, X}^{O}=1, \\
R_{X, Y}^{O} \cdot R_{Y, Z}^{O} \cdot R_{Z, X}^{O}=1 .
\end{gathered}
$$


Furthermore, if the spectral models are accurate, it should be found that

$$
R_{X, Y}^{O}=R_{X, Y}^{M} \equiv M_{X} / M_{Y} .
$$

(If $M_{X}$ is not known precisely, two measurements $R_{X, Y}^{O}$ and $R_{X, Z}^{O}$ can still be used to check the model ratio $R_{Y, Z}^{M}$ by computing $R_{Y, Z}^{O}=$ $R_{X, Z}^{O} / R_{X, Y}^{O}$.) If all three of these properties are observed, there is good reason to believe that $R_{X, Y}^{O}=R_{X, Y}^{T}$ and $O_{X, Y}=T_{X}$, though it is impossible to demonstrate the latter without absolute flux measurements.

We compute these ratios and plot them in Figure 12. Our measurements do not have enough precision for us to believably solve for spectral models of the calibrators from scratch. We do, however, solve for a change in the overall scaling of the VLA 1999.2 models, i.e., for three parameters $f_{48}, f_{147}$, and $f_{286}$ such that $M_{X}=f_{X} V_{X}$, where $V_{X}$ is the VLA model of source $X$ and we denote the calibrators by their $3 \mathrm{C}$ numbers. Given the constraints implied by Equations 1 and 2 there are only two independent $R_{X, Y}^{O}$, so a normalizing assumption must be applied to derive the three $f_{X}$ from observations. We choose to fix $f_{48} f_{147} f_{286}=1$, which is equivalent to requiring that the total relative change to the models, $\ln ^{2} f_{48}+\ln ^{2} f_{147}+\ln ^{2} f_{286}$, be minimized.

To solve for the $f_{X}$ from the data, we define $a=f_{48} / f_{147}$ and $b=f_{48} / f_{286}$. Minimizing the logarithms of $R_{X, Y}^{M} / R_{X, Y}^{O}$ in a least-squares sense leads to $a=1.018$ and $b=1.007$ with a root reduced $\chi^{2}$ parameter $\left(\sqrt{\chi_{r}^{2}}\right)$ of 1.07. This minimization included two precise measurements made at the VLA in 2008 September: $R_{48,286}^{O}=1.066 \pm$ 0.003 at $1.465 \mathrm{GHz}$ and $R_{48,286}^{O}=0.7349 \pm 0.002$ at $4.885 \mathrm{GHz}$ (R. Perley, private communication). Using our normalizing assumption, we find $f_{48}=$ 1.011, $f_{147}=0.989$, and $f_{286}=1.001$. This is consistent with evidence from the VLA that the flux density of 3C 48 has recently risen slightly (R. Perley, private communication).

Our measured flux ratios are well-described within their uncertainties by the rescaled VLA models with the exception of the $R_{147,286}^{O}$ data. These observations were made in June 2009, well after the main observing for this work was completed, after it was realized that there were only a few data comparing these calibrators. Unlike the 2008 observations, these occurred in the daytime, and solar interference was a significant problem with the low-frequency data. Nevertheless the high-frequency ratios, for which solar interference was much less relevant, disagree significantly with the adopted models. We have not been able to identify the cause of this disagreement, but we do not believe that it is due either to genuine inaccuracy in the models or to problems in the calibration and $u-v$ modeling pipeline. If the former were the case, it would represent an unprecedented variation in the flux of some combination of 3C 286 and $3 \mathrm{C} 147$. If the latter were the case, the consistency property of Equation 1 would not hold as well as it does in the observations. We believe the robustness of the rest of the results provide ample reason to believe that the models and reduction pipeline are generally robust and accurate.

\section{4. $\quad$ Spectral Modeling}

In order to assess the steepening of the nonthermal components of the observed spectra, we modeled them with a two-component model similar to that used in Niklas et al. (1997):

$$
S_{\nu}=S_{\mathrm{th}}(\nu)+S_{\mathrm{nt}}(\nu),
$$

where $S_{\mathrm{th}}(\nu)$ is the thermal contribution and $S_{\mathrm{nt}}(\nu)$ is the nonthermal contribution. Free-free absorption (FFA) is likely to play a significant role in determining the spectra of our target galaxies. We attempt to account for its effects by including as a parameter in our model $\tau_{1}$, the free-free optical depth at $1 \mathrm{GHz}$. The thermal component is then

$$
S_{\mathrm{th}}(\nu)=B_{\nu}\left(T_{e}\right) \Omega\left(1-e^{-\tau_{\mathrm{ff}}}\right),
$$

where $T_{e} \simeq 10^{4} \mathrm{~K}$ is the temperature of the thermal electrons, $\Omega$ is the angular size of the emitting region, and $\tau_{\mathrm{ff}}$ is the free-free optical depth as a function of frequency. We use the canonical frequency dependence of the free-free optical depth,

$$
\tau_{\mathrm{ff}}=\tau_{1} \nu_{g}^{-2.1},
$$

where $\nu / \mathrm{GHz}=\nu_{g}$. In typical cases, the largescale thermal emission in galaxies at $\mathrm{cm}$ wavelengths is optically thin (e.g. Duric et al. 1988; Price \& Duric 1992), $\tau_{\text {ff }} \ll 1$, in which case the thermal emission has the well-known frequency dependence of $S_{\text {th }} \propto \nu^{-0.1}$. The galaxies we consider 
are, however, decidedly atypical, with dense, compact emission regions, so we avoid this approximation in our modeling. Our results indicate, however, that it broadly holds even for the galaxies in our sample (cf. Figures 8, [11).

For simplicity we assume that the thermal and nonthermal components are well-mixed and hence share the same $\tau_{\mathrm{ff}}$. If this is not the case (as is true of the Milky Way, e.g. Beck \& Reich 1985), this assumption would require loosening in a more detailed analysis, although at small values of $\tau_{\mathrm{ff}}$ its impact is minimal. In the well-mixed case, the nonthermal emission is attenuated so that

$$
S_{\mathrm{nt}}\left(\nu_{g}\right)=\tilde{S}_{\mathrm{nt}}\left(\nu_{g}\right) \cdot \frac{1-e^{-\tau_{\mathrm{ff}}}}{\tau_{\mathrm{ff}}},
$$

where $\tilde{S}_{\text {nt }}$ is the unabsorbed nonthermal spectrum. Because our fits model the thermal and nonthermal contributions simultaneously and we do not approximate any FFA-related effects, we should obtain good results even when $\tau_{1}$ is large. We model the unabsorbed nonthermal component as

$$
\tilde{S}_{\mathrm{nt}}\left(\nu_{g}\right)=10^{A+B \log _{10} \nu_{g}+C\left[\log _{10} \nu_{g}\right]^{2} \mathrm{Jy}}
$$

a parabola in log-log space. Our parabolic spectral model is not intended to be well-motivated physically, but rather to be simple and flexible. The theoretical spectra modeled in $\mathrm{T}+06$, to which we compare our results in \$5, are derived numerically and hence do not suggest a more appropriate analytic expression for us to employ. While Condon (1992) and others typically use broken power laws to model aged synchrotron spectra, one of the key contentions of $\mathrm{T}+06$ is that other loss processes will significantly modify raw synchrotron spectra in starbursts. We note that functional forms analogous to ours have proven to be good empirical models in works such as Baars et al. (1977) and the VLA broadband calibrator models. The parabolic model has the additional benefits of being linear in its parameters (in log-space) and being reducible to a pure power-law spectrum (ignoring FFA) simply by fixing $C=0$.

An alternative model for the effect of FFA upon the nonthermal emission is one in which the absorption screens but is not mixed with the nonthermal emitting medium,

$$
S_{\mathrm{nt}}\left(\nu_{g}\right)=\tilde{S}_{\mathrm{nt}}\left(\nu_{g}\right) \cdot e^{-\tau_{\mathrm{ff}}} .
$$

We disfavor this model for our targets because they are starbursts, and their diffuse synchrotron emission propagates through a compact ISM riddled with H II regions and supernova remnants (e.g. McDonald et al. 2002; Rodríguez-Rico et al. 2004, regarding M82). In the analysis that follows we will, however, occasionally compare results for the two absorption models.

\subsection{Isolating the thermal emission}

Excepting those with active nuclei, most galaxies have a nonthermal spectrum much steeper than the aforementioned $\alpha=-0.1$ of optically-thin thermal free-free emission. Because the nonthermal emission dominates at $\mathrm{cm}$ wavelengths, good higher-frequency data are needed to usefully constrain the thermal component. However, above $\nu \simeq 100 \mathrm{GHz}$, thermal emission from cold dust begins contributing non-negligibly to the total flux. We incorporated flux densities from the literature with $20<\nu / \mathrm{GHz}<100$ into our fits for this reason. Table 6] summarizes the parameters of some of the relevant observations found in the literature. Those observations, and others not listed in table 6, are shown in Figures 8,11] for comparison to our observations.

Comparing fluxes in this way requires care because interferometers resolve out spatially extended emission. The largest angular scale (LAS) to which an interferometer observation is sensitive is inversely proportional to its shortest baseline as measured in wavelengths. In a very real way, two different interferometer observations, or an interferometer observation and a single-dish observation, are not "looking at" the same source. We deal with these considerations on a source-bysource basis below.

\subsubsection{M82}

M82 is well-observed in the $20-100 \mathrm{GHz}$ range with both interferometers and single dishes. The interferometric fluxes are systematically smaller than the single-dish fluxes by about $25 \%$, with the exception of the unusually low $87.2 \mathrm{GHz}$ singledish measurement of Jura et al. (1978). Unlike the other single-dish data, which come from maps, this observation was made with a single pointing of an $11 \mathrm{~m}$ dish (HPBW 75"). It furthermore needs conversion to the flux density scale of Baars et al. 
(1977). We regard it as an outlier and discard it.

All of the interferometer observations we consider Carlstrom \& Kronberg 1991; Rodríguez-Rico et al. 2004; Carlstrom \& Kronberg 1991; Seaquist et al. 1996) have LASs of $\sim 50^{\prime \prime}$. The deconvolved size of the radio emission of M82 is $\sim 35^{\prime \prime} \times \sim 10^{\prime \prime}$ from $\mathrm{cm}$ wavelengths (this work) up to $92 \mathrm{GHz}$ (Klein et al. 1988; Carlstrom \& Kronberg 1991), so significant flux from M82 should not be resolved out; the source of the excess emission detected by the single-dish observations is unclear. For safety, we compare the ATA fluxes only to other interferometric measurements. This criterion is coincidentally equivalent to the selection of only post-1990 observations.

\subsection{2. $N G C 253$}

The extended spatial structure of the emission of NGC 253, as detected by the ATA, makes the modeling of its spectrum more difficult. We do not expect the high-frequency interferometer observations of NGC 253 listed in Table 6] to be sensitive to the extended component because of its steep spectral index and the constrained LASs of the observations. Simple simulations of highfrequency VLA observations of the galaxy support this conclusion. Two of the FIR observations of NGC 253 (Rieke et al. 1973; Hildebrand et al. 1977) are made with single dishes with $\sim 65^{\prime \prime}$ beams that could be sensitive to the extended emission region; however, Weiß et al. (2008) resolve the central component from the extended source and find that emission from the former dominates the latter. Because of these facts, our spectra link the FIR and high-frequency measurements with fluxes for only the central component of NGC 253.

\subsubsection{Arp 220}

The observations of Arp 220 made by Imanishi et al. (2007), Norris (1988) and Zhao et al. (1996) have LASs that are too small for our purposes and are not used in our fits. The $97 \mathrm{GHz}$ singledish observation of Anantharamaiah et al. (2000) is significantly discrepant from $87 \mathrm{GHz}$ observations made with the Plateau de Bure Interferometer (Radford et al. 1991), the Nobeyama Millimeter Array (Imanishi et al. 2007), and a $110 \mathrm{GHz}$ measurement made with the Owens Valley Radio
Observatory (OVRO) mm Array (Scoville et al. 1991). The $113 \mathrm{GHz}$ measurement also presented in Anantharamaiah et al. (2000) clusters much better with these other observations and a power-law extrapolation of FIR observations of Arp 220 (Carico et al. 1992; Dunne \& Eales 2001; Eales et al. 1989; Rigopoulou et al. 1996; Woody et al. 1989). Once again, we use the interferometric observation but not the single-dish one.

\subsection{Modeling results}

We performed least-squares fits between the averaged ATA data and the chosen high-frequency measurements for each source, using a LevenbergMarquardt algorithm with $\tau_{\text {ff }}$ constrained to be nonnegative. In the case of M82 we used $\Omega=2400$ $\operatorname{arcsec}^{2}$, its modeled angular size at $1 \mathrm{GHz}$. For the core component of NGC 253, we used its modeled size as resolved at higher frequencies, 160 $\operatorname{arcsec}^{2}$. The ATA did not resolve Arp 220 but in Scoville et al. (1991) it is reported that the emission comes from an extended component of $\sim 7^{\prime \prime}$ $\times \sim 15^{\prime \prime}$ and a stronger central component of $\sim 2$ $\operatorname{arcsec}^{2}$. We adopt an intermediate value of 17 $\operatorname{arcsec}^{2}$. In all cases we used $T_{e}=10^{4} \mathrm{~K}$. The numerical results of our fits are reported in Table 7 . The uncertainties shown are marginalized values determined via Monte Carlo simulations with 10000 realizations for each source. To provide a more physical interpretation of $B$ and $C$, we also transform them, respectively, into $\alpha_{5}$, the spectral index of the nonthermal model component at $5 \mathrm{GHz}$, and $\Delta \equiv \mathrm{d} \alpha / \mathrm{d}\left(\log _{10} \nu\right)$ at the same frequency. We also compute $f_{\mathrm{th}}$, the thermal fraction (not correcting for FFA) at $1 \mathrm{GHz}$. We computed $3 \sigma$ confidence limits in the total flux of the best-fit models at 40 frequencies logarithmically spaced between 0.7 and $100 \mathrm{GHz}$. Finally, we computed $1 \sigma$ confidence regions in the $B-C$ plane of parameter space. This was done by tracing the contour of $\chi^{2}=\chi_{\min }^{2}+2.3$, with $\chi_{\min }^{2}$ being the minimum $\chi^{2}$ achieved in the overall fit and the offset parameter coming from Avni (1976). The value of $\chi^{2}$ assigned to each location in the $B-C$ plane is the minimum value of $\chi^{2}$ attained in a fit with $B$ and $C$ fixed and $A$ and $\tau_{1}$ free.

The parameter values that are obtained depend strongly on the highest-frequency data points. Fits that were run without any very 
high-frequency $(\nu>50 \mathrm{GHz})$ data points often converged to the physically-suspect lower bound of $\tau_{1}=0$. To investigate the possible effects of inaccurate high-frequency observations on our results, we ran a set of Monte Carlo simulations in which the uncertainty associated with each high-frequency measurement was doubled. In this case, the resulting uncertainties in the modeled parameter values increased by a factor of $\sim 1.5$, demonstrating the extent to which the results are affected by those few data points. While we have done our best to choose our high-frequency data points carefully, it remains true that our results are dependent on the choice of which measurements to include and, of course, the accuracy of those measurements.

\subsubsection{M82}

The M82 fit has $\sqrt{\chi_{r}^{2}}=1.21$. The ATA data and fit results are presented in Figure 8 . The value of $\alpha_{5}$ that we obtain agrees well with that found in Klein et al. (1988), $\alpha=-0.68 \pm 0.02$. We, however, find a higher thermal content at $32 \mathrm{GHz}$, because the possibility of a steepening nonthermal spectrum allows for a larger thermal contribution.

A fit with a fixed value of $C=0$ (i.e., a pure power-law nonthermal spectrum) had a comparable $\sqrt{\chi_{r}^{2}}=1.34$, but also $\tau_{1}=0$, a physically implausible result. In the $C=0$ case, the many ATA data points tightly constrain the power-law slope of the nonthermal component, but the highfrequency points lie significantly below the nonthermal line. The thermal component, being spectrally shallower than the nonthermal component, can only make this situation worse, so the fit converges to a solution with no thermal component at all. If any thermal component is present, the nonthermal spectrum must steepen so that the highfrequency points can be modeled successfully.

\subsection{2. $N G C 253$}

The NGC 253 fit has $\sqrt{\chi_{r}^{2}}=1.02$. The ATA data and model results for the core component of NGC 253 are presented in Figure 9. The symbols used are analogous to those in Figure 8, Figure 10 shows the extended and total fluxes in context with total-flux data from the literature. The same models as in Figure 9 are overlaid to ease comparison.
A fit with a fixed value of $C=0$ had $\sqrt{\chi_{r}^{2}}=$ 1.51 and $\tau_{1}=0.25$. Here, a change in the spectral index is detectable in the ATA data alone, so when the nonthermal component has a constant slope, $\tau_{1}$ must increase to induce more curvature at low frequencies via FFA. In this case, once again the high-frequency data points lie well below the points attained by the model.

\subsubsection{Arp 220}

The Arp 220 fit has $\sqrt{\chi_{r}^{2}}=1.23$ and $\tau_{1}=0$ and is shown in Figure 11. This result is due to the dramatic steepening of the spectrum of Arp 220 at $\nu \sim 10 \mathrm{GHz}$. A purely nonthermal fit that accommodates the high-frequency observations must underestimate the low-frequency $(\nu \lesssim 2 \mathrm{GHz})$ data points significantly. The addition of any thermal contribution would require an even steeper nonthermal component to match the high-frequency measurements, which would make the discrepancies on the low end even worse. Adjustments to the assumed angular size of Arp 220, which affect the overall scaling of the thermal component, do not improve the outcome. We report the results of our fit in Table 7 but caution that they are unsatisfactory. We exclude the Arp 220 results from further analysis in the following sections.

If the nonthermal curvature parameter $C$ is fixed to zero, the model cannot accommodate the data, with $\sqrt{\chi_{r}^{2}}=2.77$. This result quantitatively confirms the supposition of Condon (1992) that the nonthermal spectrum of Arp 220 steepens in the $\mathrm{cm}$ regime. Our data cause serious problems for the empirical spectral model of Arp 220 presented in Sopp \& Alexander (1991), which predicts a much steeper spectrum at frequencies of a few $\mathrm{GHz}$ than we find. Their model was inconsistent with several measurements at $\nu \sim 2 \mathrm{GHz}$ shown in the paper, and our ATA data make it clear that those measurements are generally accurate and the simple power-law used in that work is insufficient to match the data.

\section{Discussion}

We test whether our observational results for M82 and NGC 253 are consistent with the predictions of $\mathrm{T}+06$ by comparing the curvature of the FFA-corrected nonthermal spectra with the output of the numerical model presented in that work. 
We obtained an updated version of the relevant code (E. Quataert, private communication) and modified it to allow more convenient tuning of the input parameters. These parameters are (1) $\Sigma_{g}$, the surface density of the gas disk; (2) $h_{\mathrm{eff}}$, the effective scale height of the gas disk; (3) $p$, the power-law index of the injected CR energy spectrum, $\mathrm{d} n \propto E^{-p} \mathrm{~d} E$; and (4) $\eta$, the ratio of the energy density of the magnetic field to the pressure of the ISM, $B^{2}=\eta \cdot 8 \pi^{2} G \Sigma_{g}^{2}$. The value of $h_{\text {eff }}$ affects the characteristic timescales of CR cooling via ionization and free-free losses by setting the density of the neutral ISM as encountered by the CRs, $n_{\mathrm{CR}}=\Sigma_{g} / 2 m_{p} h_{\text {eff }}$. (In T+06, $h_{\text {eff }}$ is specified by two parameters, the physical scale height of the gas disk $h$ and an ISM/CR interaction efficiency parameter $f: h_{\mathrm{eff}}=h / f$. They are degenerate for our purposes.) Because the mean value of the ISM density may not correspond well to the value of $n_{\mathrm{CR}}$ due to clumping and other effects, $h_{\text {eff }}$ may likewise not correspond well to the actual scale height of the gas disk. We take the surface density of the disk of M82 to be $0.081 \mathrm{~g} \mathrm{~cm}^{-2}$ and that of NGC 253 to be $0.067 \mathrm{~g} \mathrm{~cm}^{-2}$ (Thompson et al. 2009, and references therein). Note that these recently-derived values are significantly different than the ones given in Kennicutt (1998).

Given a choice of $\Sigma_{g}, h_{\text {eff }}, p$, and $\eta$, we used a simple least-squares fit to determine values for the spectral parameters $B$ and $C$. We used the $\mathrm{T}+06$ model to compute flux densities at 25 frequencies logarithmically spaced between 1 and $7 \mathrm{GHz}$ and then fit a bent power law (as in Equation 8) to the data. All of the spectra generated in this way were very well-described by such a model. The derived parameters were not sensitive to the details of the choice of frequencies at which to sample the model.

We computed such $B$ and $C$ values for the $\mathrm{T}+06$ model on two grids of parameters. The first had $\Sigma_{g}$ set to the observed M82 value, $h_{\text {eff }}$ ranging between 10 and $250 \mathrm{pc}, p$ ranging between 2 and 2.5 , and $\eta$ ranging between 0.02 and 1 , all stepping through 10 values logarithmically. The lower bound of $\eta$ is the approximate value for which the synchrotron cooling time, $\tau_{\text {syn }}$, and the timescale for escape from the galaxy via advection by a galactic wind, $\tau_{\text {esc }}$, are equal. For $\eta$ (and hence $B$ ) smaller than this, the assumptions of the $\mathrm{T}+06$ code are no longer upheld. The largest plausible $\eta$ is $\sim 1$, at which point the magnetic field and
ISM pressure are in equipartition (Parker 1966). The second grid was identical except that $\Sigma_{g}$ was set to the NGC 253 value and the lower bound of $\eta$ was 0.04 , the analogous limiting value. Figure 13 shows the values of $B$ and $C$ attained by the model and compares them to the $1 \sigma$ and $2 \sigma$ confidence intervals associated with our observations. The $\mathrm{T}+06$ models can accommodate a wide range of spectral indices and exhibit spectral steepening for all parameter values. Our observed spectra, however, exhibit more steepening (more negative $C)$ than the models. The models are consistent with observations of both galaxies at the $2 \sigma$ level but only with M82 at the $1 \sigma$ level. If the source of the FFA is configured as a screen in front of the nonthermal emission rather than a medium mixed within it (Equation 9), the $C$ values derived for our observations are less negative, $\sim-0.10$, and the $\mathrm{T}+06$ models have large areas of overlap with the observations of both galaxies even at the $1 \sigma$ level. The $\sqrt{\chi_{r}^{2}}$ values of the fits in this case are virtually identical to those of the fits in the wellmixed case.

We quantify this agreement in Figures 14 and 15. which show the regions in the $\eta$ - $h_{\text {eff }}$ plane where the results of the $\mathrm{T}+06$ model are consistent with the observed properties of M82 and NGC 253, respectively, at the $2 \sigma$ level, for $p=2$ and 2.3. Because our data, unlike the outputs of the $\mathrm{T}+06$ models, are affected by FFA and thermal emission, they cannot be compared to the models directly without taking into account these effects. To do so requires some sort of spectral modeling, so we compare the spectral curvature parameters $B$ and $C$ found for our data with ones derived for the outputs of the $\mathrm{T}+06$ models. (If we corrected our observations for the modeled FFA and thermal emission and then compared them to the $\mathrm{T}+06$ models, we would be effectively hiding the model-dependence of the correction and discarding the information that the data have been corrected assuming an underlying parabolic nonthermal spectral model.) We assumed a bivariate normal probability distribution for the observed values of $B$ and $C$, with the parameters of the distribution estimated by applying the maximum likelihood method to the results of the Monte Carlo simulations. The agreement between the shape of the analytic distribution function and contours of constant $\Delta \chi^{2}$ was very good. Using the bounds on 
$h_{\text {eff }}$ and $\eta$ given above, $p=2.5$ is rejected at the $99.96 \%$ confidence limit for M82 and the $99.99 \%$ confidence limit for NGC 253; larger values of $p$ become acceptable if the lower limit on $h_{\text {eff }}$ is decreased. For NGC 253, $p=2.3$ is also rejected at the $99.99 \%$ confidence limit.

For M82, virtually all physically plausible values of $\eta$ are allowed. For $p=2$, the allowed values of $h_{\text {eff }}$ are of order $100 \mathrm{pc}$, broadly consistent with observed radio sizes. For $p=2.3$, agreement requires $h_{\mathrm{eff}} \approx 20 \mathrm{pc}$. Such sizes have been proposed for the neutral gas disks of some ultraluminous infrared galaxies (ULIRGs; Scoville et al. 1997; Carilli et al. 1998), but are unlikely to be found in less extreme galaxies. Furthermore, if the CRs interact with the ISM at somewhat less than its nominal mean density (the parameter $f$ of $\mathrm{T}+06$ is $<1$ ), as is suggested by measurements of CRs in the Milky Way (Connell 1998), the actual scale height of the disk becomes even less physically plausible. As $\eta$ increases, the allowed $h_{\text {eff }}$ decreases for both values of $p$. This is because the stronger magnetic field steepens the synchrotron spectrum, requiring a smaller $h_{\text {eff }}$ to increase $n_{\mathrm{CR}}$ and consequently the amount of spectral flattening due to ionization and free-free emission off of neutral hydrogen. As $p$ increases, the allowed values of $h_{\text {eff }}$ rapidly decrease for the same reason.

The NGC 253 data are only marginally consistent with the $\mathrm{T}+06$ model within the range of parameters we consider, and the statistically acceptable results have $h_{\mathrm{eff}} \approx 50 \mathrm{pc}$. If the source of FFA is configured as a screen rather than mixed with the nonthermal emission, much more of the $\mathrm{T}+06$ model parameter space is allowed, with a general configuration of allowed parameters similar to that obtained for M82.

The relationship between $h_{\mathrm{eff}}$ and the observed radio sizes of starburst galaxies $h_{\mathrm{obs}}$ is significant within the $\mathrm{T}+06$ model. Estimates in $\mathrm{T}+06$ indicate that if $h_{\mathrm{eff}} \sim h_{\mathrm{obs}}$ and $B \sim B_{\mathrm{min}}$, free-free emission off of neutral ISM nuclei becomes a significant sink of CR energy for $\Sigma_{g} \gtrsim 1 \mathrm{~g} \mathrm{~cm}^{-2}$, which would disrupt the observed linearity of the FIRradio correlation at high surface densities. Since the free-free timescale is independent of the magnetic field while the synchrotron timescale goes as $\tau_{\text {syn }} \propto B^{-3 / 2}$, a stronger magnetic field, $B \sim B_{\text {eq }}$, avoids this inconsistency. Our observations have $h_{\mathrm{eff}} \sim h_{\mathrm{obs}}$ in the $p=2$ case and $h_{\mathrm{eff}} \ll h_{\mathrm{obs}}$ in the $p=2.3$ case. A precise independent measurement of $p$ could thus provide a valuable test of this aspect of the $\mathrm{T}+06$ model.

\section{Conclusions}

Our observations of M82 are consistent with the scenario proposed in $\mathrm{T}+06$. Our observations of NGC 253, on the other hand, are only marginally consistent with it. We decisively detect the spectral steepening at $\nu \simeq 1 \mathrm{GHz}$ predicted in that work. The uncertainties in our spectral modeling, due to uncertainties in the individual observations, the difficulties of untangling the thermal and nonthermal emission, and the inaccuracies inherent in our spatially-unresolved approach, make it difficult to significantly constrain all of the parameters of the $\mathrm{T}+06$ spectral model or to reject its entire parameter space. We do, however, reject with high confidence the $\mathrm{T}+06$ model with a steep electron energy injection spectrum power-law index of $p=2.5$ for M82 and NGC 253. We also reject with high confidence the $\mathrm{T}+06$ model with $p=2.3$ for NGC 253. We are unable to place limits on the strength of the magnetic field required by the $\mathrm{T}+06$ model with our current data. Further work to constrain the efficiency with which CRs interact with the ISM or the allowed values of $p$ would significantly shrink the model parameter space. Observations of a larger sample of galaxies would allow a statistical treatment of the topics that we have dealt with here on a case-by-case basis. It would likely also be fruitful to compare our detailed radio results with FIR observations. Such an approach would be a frequency-space analog to the recent works investigating the connections between FIR and radio emission at high spatial resolutions (Marsh \& Helou 1995; Paladino et al. 2006).

The broadband, continuous spectra that we have obtained with the ATA are precise and repeatable. The quality of our measurements is limited by several factors. The $u-v$ coverage of our snapshot observations is good but suboptimal for complex sources, which made the imaging of targets such as NGC 253 more difficult. The sensitivity the system is a limiting factor for snapshot observations of fainter sources such as Arp 220. Both of these quantities were limited by the limited number of antennas available for observing during 
the commissioning of the ATA-42. The planned expansion of the ATA to $\sim 350$ antennas will do much to improve both of them. The fact that the ATA was undergoing commissioning at the time of these observations meant that several important aspects of observing and data reduction, such as polarization calibration and RFI mitigation, were incomplete. Ongoing development since these observations were made has led to improvements in virtually all aspects of the system performance. Of particular interest is the deployment of a second independently-tunable correlator, which doubles the already-fast throughput of our spectral observations and further improves the prospects for fast, precise, broadband spectral monitoring of sources.

The authors would like to acknowledge the generous support of the Paul G. Allen Family Foundation, who have provided major support for design, construction, and operations of the ATA. Contributions from Nathan Myhrvold, Xilinx Corporation, Sun Microsystems, and other private donors have been instrumental in supporting the ATA. The ATA has been supported by contributions from the US Naval Observatory in addition to National Science Foundation grants AST-050690 and AST-0838268.

Our work would not have been possible if not for the tireless efforts of everyone on the ATA team: Jack Welch, Don Backer, Leo Blitz, Douglas Bock, Calvin Cheng, Steve Croft, Matt Dexter, Greg Engargiola, Ed Fields, R. James Forster, Colby Gutierrez-Kraybill, Carl Heiles, Tamara Helfer, Susanne Jorgensen, Garrett Keating, Casey Law, John Lugten, Dave MacMahon, Peter McMahon, Oren Milgrome, Andrew Siemion, Douglas Thornton, Lynn Urry, Joeri van Leeuwen, Dan Werthimer, Melvyn Wright; Jill Tarter, Rob Ackermann, Shannon Atkinson, Peter Backus, Billy Barott, Tucker Bradford, Mike Davis, Dave DeBoer, John Dreher, Gerry Harp, Jane Jordan, Tom Kilsdonk, Tom Pierson, Karen Randall, John Ross, Seth Shostak, Ken Smolek; Matt Fleming, Chris Cork, Artyom Vitouchkine; Niklas Wadefalk, and Sandy Weinreb.

We thank Eliot Quataert for helpful discussions. We are indebted to the numerous programmers and scientists who have contributed to the software systems used in this work, the entirety of which are open-source and freely available for inspection and use by anyone in the world. This research has made use of NASA's Astrophysics Data System; the SIMBAD database, operated at CDS, Strasbourg, France; and the NASA/IPAC Extragalactic Database (NED) which is operated by the Jet Propulsion Laboratory, California Institute of Technology, under contract with the National Aeronautics and Space Administration. PKGW is supported by an NSF Graduate Research Fellowship.

\section{REFERENCES}

Anantharamaiah, K. R., Viallefond, F., Mohan, N. R., Goss, W. M., \& Zhao, J.-H. 2000, ApJ, 537,613 .

Appleton, P. N., Fadda, D. T., Marleau, F. R., Frayer, D. T., Helou, G., Condon, J. J., Choi, P. I., Yan, L., Lacy, M., Wilson, G., Armus, L., Chapman, S. C., Fang, F., Heinrichson, I., Im, M., Jannuzi, B. T., Storrie-Lombardi, L. J., Shupe, D., Soifer, B. T., Squires, G., \& Teplitz, H. I. 2004, ApJS, 154, 147.

Avni, Y. 1976, ApJ, 210, 642.

Baars, J. W. M., Genzel, R., Pauliny-Toth, I. I. K., \& Witzel, A. 1977, A\&A, 61, 99.

Backer, D. 2007, CASPER Memo series \#14.

Beck, R., Biermann, P., Emerson, D. T., \& Wielebinski, R. 1979, A\&A, 77, 25.

Beck, R. \& Reich, W. 1985, in IAU Symposium 106, The Milky Way Galaxy, ed. H. van Woerden, R. J. Allen, \& W. Butler-Burton (Dordrecht: Reidel), p. 239.

Blandford, R., \& Eichler, D. 1987, Phys. Rep., 154,1 .

Boulanger, F., \& Pérault, M. 1988, ApJ, 330, 964.

Breitschwerdt, D., McKenzie, J. F., \& Völk, H. J. 1991, A\&A, 245, 79.

Burbidge, G. R. 1956, ApJ, 124, 416.

Byrd, R. H., Lu, P., \& Nocedal, J. 1995, SIAM Journal on Scientific and Statistical Computing, 16, 1190. 
Carico, D. P., Keene, J., Soifer, B. T., \& Neugebauer, G. 1992, PASP, 104, 1086.

Carilli, C. L., Wrobel, J. M., \& Ulvestad, J. S. 1998, AJ, 115, 928.

Carlstrom, J. E., \& Kronberg, P. P. 1991, ApJ, $366,422$.

Clark, B. G. 1980, A\&A, 89, 3.

Condon, J. J. 1992, ARA\&A, 30, 575.

Condon, J. J., Cotton, W. D., Greisen, E. W., Yin, Q. F., Perley, R. A., Taylor, G. B., \& Broderick, J. J. 1998, AJ, 115, 1693.

Connell, J. J. 1998, ApJ, 501, L59.

Conway, J. E., Cornwell, T. J., \& Wilkinson, P. N. 1990, MNRAS, 246, 490.

Cox, M. J., Eales, S. A. E., Alexander, P., \& Fitt, A. J. 1988, MNRAS, 235, 1227.

de Jong, T., Klein, U., Wielebinski, R., Wunderlich, E. 1985, A\&A, 147, L6.

Devereux, N. A., \& Eales, S. A. 1989, ApJ, 340, 708.

Downes, D., \& Solomon, P. M. 1998, ApJ, 507, 615.

Dunne, L., \& Eales, S. A. 2001, MNRAS, 327, 697.

Duric, N., Bourneuf, E., \& Gregory, P. C. 1988, AJ, 96, 81.

Eales, S. A., Wynn-Williams, C. G., \& Duncan, W. D. 1989, ApJ, 339, 859.

Fitt, A. J., Alexander, P., \& Cox, M. J. 1988, MNRAS, 233, 907.

Geldzahler, B. J., \& Witzel, A. 198, AJ, 86, 1306.

Harwit, M., \& Pacini, F. 1975, ApJ, 200, L127

Helou, G., Soifer, B. T., \& Rowan-Robinson, M. 1985, ApJ, 298, L7.

Hildebrand, R. H., Whitcomb, S. E., Winston, R., Stiening, R. F., Harper, D. A., \& Moseley, S. H. 1977, ApJ, 216, 698.

Högbom, J. A. 1974, A\&AS, 15, 417.
Hummel, E., Smith, P., \& van der Hulst, J. M. 1984, A\&A, 137, 138.

Hummel, E., Davies, R. D., Wolstencroft, R. D., van der Hulst, J. M., \& Pedlar, A. 1988, A\&A, 199, 91.

Imanishi, M., Nakanishi, K., Tamura, Y., Oi, N., \& Kohno, K. 2007, AJ, 134, 2366.

Jura, M., Hobbs, R. W., \& Maran, S. P. 1978, AJ, $83,153$.

Kennicutt, R. 1998, ApJ, 498, 541.

Klein, U., Wielebinski, R., \& Morsi, H. W. 1988, A\&A, 190, 41.

Kronberg, P. P., Emerson, D. T., Klein, U., \& Wielebinski, R. 1979, ApJ, 230, L149.

Leeuw, L. L., Robson, I. E. 2009, AJ, 137, 517.

Lisenfeld, U., Völk, H. J., Xu, C. 1996, A\&A, 306, 677.

Longair, M. S. 1994, High Energy Astrophysics (2nd ed.; Cambridge: Cambridge Univ. Press).

Marsh, K. A., \& Helou, G. 1995, ApJ, 445, 599.

McDonald, A. R., Muxlow, T. W. B., Wills, K. A., Pedlar, A., \& Beswick, R. J. 2002, MNRAS, 334, 912.

Niklas, S., Klein, U., Braine, J., \& Wielebinski, R. 1995, A\&AS, 114, 21.

Niklas, S., Klein, U., \& Wielebinski, R. 1997, A\&A, 322, 19.

Norris, R. P. 1988, MNRAS, 230, 345.

Ott, J., Weiß, A., Henkel, C., Walter, F. 2005, ApJ, 629, 767.

Paladino, R., Murgia, M., Helfer, T. T., Wong, T., Ekers, R., Blitz, L., Gregorini, L., \& Moscadelli, L. 2006, A\&A, 456, 847.

Parker, E. N. 1966, ApJ, 145, 811.

Peng, R., Zhou, S., Whiteoak, J. B., Lo, K. Y., \& Sutton, E. C. 1996, ApJ, 470, 821.

Price, R., \& Duric, N. 1992, ApJ, 401, 81. 
Radford, S. J. E., Delannoy, J., Downes, D., Guélin, M., Guilloteau, S., Greve, A., Lucas, R., Morris, D., \& Wink, J. 1991, Dynamics of Galaxies and Their Molecular Cloud Distributions, ed. F. Combes \& F. Casoli (Kluwer: Dordrecht), 146, 303.

Ricci, R., Prandoni, I., Gruppioni, C., Sault, R. J., \& de Zotti, G. 2006, A\&A, 445, 465.

Rieke, G. H., Harper, D. A., Low, F. J., Armstrong, K. R. 1973, ApJ, 183, L67.

Rigopoulou, D., Lawrence, A., \& RowanRobinson, M. 1996, MNRAS, 278, 1049.

Rodríguez-Rico, C. A., Viallefond, F., Zhao, J.-H., Goss, W. M., \& Anantharamaiah, K. R. 2004, ApJ, 616, 783.

Rodríguez-Rico, C. A., Goss, W. M., Viallefond, F., Zhao, J.-H., Gómez, Y., \& Anantharamaiah, K. R. 2005, ApJ, 633, 198.

Sault, R. J., Teuben, P. J., \& Wright, M. C. H. 1995, in ASP Conf. Ser. 77, A Retrospective View of Miriad, Astronomical Data Analysis Software and Systems IV, ed. R. A. Shaw, H. E. Payne, \& J. J. E. Hayes (San Francisco, CA: ASP), 433.

Scoville, N. Z., Soifer, B. T., Neugebauer, G., Young, J. S., Matthews, K., \& Yerka, J. 1985, ApJ, 289, 129.

Scoville, N. Z., Sargent, A. I., Sanders, D. B., \& Soifer, B. T. 1991, ApJ, 366, L5.

Scoville, N. Z., Yun, M. S., \& Bryant, P. M. 1997, ApJ, 484, 702.

Seaquist, E. R., Bell, M. B., \& Bignell, R. C. 1985, ApJ, 294, 546.

Seaquist, E. R., Carlstrom, J. E., Bryant, P. M., \& Bell, M. B. 1996, ApJ, 465, 691.

Sopp, H. M., \& Alexander, P. 1991, MNRAS, 251, 112.

Steer, D. G., Dewdney, P. E., \& Ito, M. R. 1984, A\&A, 137, 2.

Strickland, D. K., Heckman, T. M., Colbert, E. J. M., Hoopes, C. G., Weaver, K. A. 2004, ApJ, 606, 829.
Takano, S., Hofner, P., Winnewisser, G., Nakai, N., \& Kawaguchi, K. 2005, PASJ, 57, 549.

Thompson, T. A., Quataert, E., Waxman, E., Murray, N., \& Martin, C. L. 2006, ApJ, 645, $186(\mathrm{~T}+06)$.

Thompson, T. A., Quataert, E., \& Murray, N. 2009, MNRAS, 397, 1410.

van der Kruit, P. C. 1971, A\&A, 15, 110.

Völk, H. J. 1989, A\&A, 218, 67.

Walterbos, R. A. M., \& Schwering, P. B. W. 1987, A\&A, 180, 27.

Weiß, A., Kovács, A., Güsten, R., Menten, K. M., Schuller, F., Siringo, G., \& Kreysa, E. 2008, A\&A, 490, 77.

Welch, W. J., Backer, D., Blitz, L., Bock, D., Bower, G. C., Cheng, C., Croft, S., Dexter, M., Engargiola, G., Fields, E., Forster, R., Gutierrez-Kraybill, C., Heiles, C., Helfer, T., Jorgensen, S., Keating, G., Lugten, J., MacMahon, D., Milgrome, O., Thornton, D., Urry, L., van Leeuwen, J., Werthimer, D., Williams, P. H., Wright, M., Tarter, J., Ackermann, R., Atkinson, S., Backus, P., Barott, W., Bradford, T., Davis, M., DeBoer, D., Dreher, J., Harp, G., Jordan, J., Kilsdonk, T., Pierson, T., Randall, K., Ross, J., Shostak, S., Fleming, M., Cork, C., Vitouchkine, A., Wadefalk, N., \& Weinreb, S. 2009, Proc. IEEE, 97, 1438.

Woody, D. P., Scott, S. L., Scoville, N. Z., Mundy, L. G., Sargent, A. I., Padin, S., Tinney, C. G., Wilson, C. D. 1989, ApJ, 337, L41.

Wright, A., \& Otrupcek, R. 1990, Parkes Catalog (PKS Catalog: Australia Telescope National Facility)

Wunderlich, E., \& Klein, U. 1988, A\&A, 206, 47.

Yun, M. S., Reddy, N. A., Condon, J. J. 2001, ApJ, 554, 803.

Yun, M. S., \& Carilli, C. L. 2002, ApJ, 568, 88.

Zhao, J.-H., Anantharamaiah, K. R., Goss, W. M., \& Viallefond, F. 1996, ApJ, 472, 54. 
Zhu, C., Byrd, R. H., \& Nocedal, J. 1997, ACM

Transactions on Mathematical Software, 23, 550.

This 2-column preprint was prepared with the AAS LATEX macros v5.2. 
TABLE 1

OBSERVATIONS

\begin{tabular}{|c|c|c|c|}
\hline Date (UT) & Segment \# & Sources & Frequencies (GHz) \\
\hline 20080912 & 1 & 3C 48, 3C 147, M82, NGC 253 & $\begin{array}{l}1.4,1.5,2.0,2.1,3.1,4.0^{\mathrm{a}}, 4.1^{\mathrm{a}}, \\
\quad 5.0,5.1\end{array}$ \\
\hline \multirow[t]{4}{*}{20080913} & 2 & 3C 286, Arp 220 & $1.5,1.6,2.5,2.6,4.5,4.6$ \\
\hline & 3 & $3 \mathrm{C} 147$, NGC 253 & $\begin{array}{l}1.1,1.2,1.3,2.6,2.7,3.6,3.7^{\mathrm{a}}, 5.6 \text {, } \\
\quad 5.7\end{array}$ \\
\hline & 4 & 3C 48, 3C 147, M82 & $3.2,3.3,4.4,4.5,5.5,5.6,6.6,6.7$ \\
\hline & 5 & 3C 286, Arp 220 & $1.1,1.2,2.5,2.6,5.0,5.7,6.3$ \\
\hline \multirow[t]{4}{*}{20080914} & 6 & 3C 48, Arp 220 & $1.1,1.2,2.5,2.6,5.0,5.7,6.3$ \\
\hline & 7 & 3C 147, NGC 253 & $2.7,3.1,3.3,4.5,4.7,6.1,6.3$ \\
\hline & 8 & 3C 48, 3C 147, M82 & $1.0,1.1,2.6,2.9,4.8,5.0$ \\
\hline & 9 & 3C 286, Arp 220 & $1.9,2.9,3.2,3.3,5.4$ \\
\hline 20080924 & 10 & 3C 286, Arp 220, M82 & $\begin{array}{l}1.4,1.7,1.8,2.0,3.4,3.5,3.6,5.0 \\
\quad 5.2,6.0\end{array}$ \\
\hline \multirow[t]{4}{*}{20081015} & 11 & $3 \mathrm{C} 48,3 \mathrm{C} 286, \operatorname{Arp} 220$ & $\begin{array}{l}0.5,0.7,0.9,1.4,4.3,4.4,4.7,5.0 \\
\quad 6.2,6.7,7.0\end{array}$ \\
\hline & 12 & 3C 48, 3C 147, M82 & $1.4,2.4,2.5,4.3,4.7,5.0$ \\
\hline & 13 & 3C 147, NGC 253 & $\begin{array}{l}1.4,1.7,1.8,1.9,4.5,4.6,4.9,5.0 \\
\quad 5.8,5.9,6.0,6.4,6.7,7.0\end{array}$ \\
\hline & 14 & 3C 48, 3C 147, M82 & $6.1,6.2,6.3,6.4$ \\
\hline 20090307 & 15 & $3 \mathrm{C} 48,3 \mathrm{C} 286$ & $1.4,3.1,5.0,6.0,7.0$ \\
\hline 20090621 & 16 & 3C $147,3 \mathrm{C} 286$ & $\begin{array}{l}1.0,1.4,1.5,5.0,5.1,5.5,6.1,6.5 \text {, } \\
\quad 7.0\end{array}$ \\
\hline
\end{tabular}

${ }^{a}$ Discarded due to severe RFI. 
TABLE 2

SNAPShots MADE DURING SEGMENT \#12

\begin{tabular}{cccccc}
\hline \hline $\begin{array}{c}\text { Time } \\
(\mathrm{UT})\end{array}$ & Pointing $^{\mathrm{a}}$ & $\begin{array}{c}\text { Azimuth } \\
(\mathrm{deg})\end{array}$ & $\begin{array}{c}\text { Elevation } \\
(\mathrm{deg})\end{array}$ & $\begin{array}{c}\nu_{\text {sky }} \\
(\mathrm{GHz})\end{array}$ & $\begin{array}{c}\text { Focus }^{\mathrm{a}} \\
(\mathrm{GHz})\end{array}$ \\
\hline $03: 50$ & $3 \mathrm{C} 48$ & 77 & 39 & 1.4 & 2.0 \\
$03: 53$ & & 77 & 40 & 2.4 & 3.0 \\
$03: 55$ & & 78 & 40 & 2.5 & \\
$03: 59$ & M82 & 359 & 21 & 1.4 & 2.0 \\
$04: 02$ & & 358 & 21 & 2.4 & 3.0 \\
$04: 04$ & & 358 & 21 & 2.5 & \\
$04: 08$ & $3 \mathrm{C} 48^{\mathrm{b}}$ & 79 & 43 & 4.3 & 5.0 \\
$04: 11$ & & 80 & 43 & 4.7 & \\
$04: 13$ & & 80 & 44 & 5.0 & \\
$04: 17$ & M82 & 359 & 21 & 4.3 & \\
$04: 20$ & & 0 & 21 & 4.7 & \\
$04: 22$ & & 0 & 20 & 5.0 & \\
$04: 28$ & $3 \mathrm{C} 48^{\mathrm{b}}$ & 82 & 47 & 1.4 & 2.0 \\
$04: 31$ & & 82 & 47 & 2.4 & 3.0 \\
$04: 34$ & & 83 & 48 & 2.5 & \\
$04: 38$ & M82 & 1 & 21 & 1.4 & 2.0 \\
$04: 41$ & & 2 & 21 & 2.4 & 3.0 \\
$04: 43$ & & 2 & 21 & 2.5 & \\
$04: 47$ & $3 \mathrm{C} 147$ & 40 & 19 & 1.4 & 2.0 \\
$04: 50$ & & 40 & 19 & 2.4 & 3.0 \\
$04: 52$ & & 40 & 20 & 2.5 & \\
$04: 56$ & $3 \mathrm{C} 48$ & 86 & 52 & 4.3 & 5.0 \\
$04: 58$ & & 86 & 52 & 4.7 & \\
$05: 01$ & & 87 & 53 & 5.0 & \\
$05: 04$ & M82 & 4 & 21 & 4.3 & \\
$05: 07$ & & 4 & 21 & 4.7 & \\
$05: 09$ & & 4 & 21 & 5.0 & \\
\hline
\end{tabular}

${ }^{a} \mathrm{~A}$ blank entry indicates no change from the previous value.

${ }^{\mathrm{b}} \mathrm{Had}$ it been above the $18^{\circ}$ elevation limit of the ATA, 3C 147 would have been observed at these times. 
TABLE 3

Flux DEnsity Measurements of M82

\begin{tabular}{|c|c|c|}
\hline$\nu(\mathrm{GHz})$ & Flux (Jy) & Calibrator \\
\hline \multirow[t]{2}{*}{1.0} & $8.92 \pm 0.27$ & 3C 48 \\
\hline & $8.96 \pm 0.16$ & 3C 147 \\
\hline \multirow[t]{2}{*}{1.1} & $8.72 \pm 0.26$ & $3 \mathrm{C} 48$ \\
\hline & $8.46 \pm 0.15$ & 3C 147 \\
\hline \multirow[t]{2}{*}{1.4} & $7.25 \pm 0.11$ & $3 \mathrm{C} 48$ \\
\hline & $7.37 \pm 0.10$ & 3C 286 \\
\hline 1.5 & $6.85 \pm 0.15$ & 3C 48 \\
\hline 1.7 & $6.60 \pm 0.09$ & 3C 286 \\
\hline 1.8 & $6.34 \pm 0.09$ & 3C 286 \\
\hline \multirow[t]{2}{*}{2.0} & $6.06 \pm 0.13$ & $3 \mathrm{C} 48$ \\
\hline & $6.13 \pm 0.08$ & $3 \mathrm{C} 286$ \\
\hline 2.1 & $5.87 \pm 0.13$ & $3 \mathrm{C} 48$ \\
\hline 2.5 & $5.39 \pm 0.12$ & $3 \mathrm{C} 48$ \\
\hline \multirow[t]{2}{*}{2.6} & $5.55 \pm 0.17$ & $3 \mathrm{C} 48$ \\
\hline & $5.21 \pm 0.09$ & 3C 147 \\
\hline \multirow{2}{*}{2.9} & $4.89 \pm 0.11$ & $3 \mathrm{C} 48$ \\
\hline & $4.88 \pm 0.11$ & 3C 147 \\
\hline 3.0 & $4.33 \pm 0.09$ & $3 \mathrm{C} 48$ \\
\hline 3.1 & $4.70 \pm 0.10$ & $3 \mathrm{C} 48$ \\
\hline 3.2 & $4.54 \pm 0.07$ & 3C 147 \\
\hline 3.3 & $4.50 \pm 0.07$ & 3C 147 \\
\hline 3.4 & $4.44 \pm 0.06$ & 3C 286 \\
\hline 3.5 & $4.32 \pm 0.06$ & 3C 286 \\
\hline 3.6 & $4.15 \pm 0.06$ & 3C 286 \\
\hline 4.3 & $3.85 \pm 0.09$ & $3 \mathrm{C} 48$ \\
\hline 4.4 & $3.73 \pm 0.06$ & 3C 147 \\
\hline 4.5 & $3.67 \pm 0.06$ & 3C 147 \\
\hline 4.7 & $3.55 \pm 0.08$ & $3 \mathrm{C} 48$ \\
\hline \multirow[t]{2}{*}{4.8} & $3.53 \pm 0.08$ & $3 \mathrm{C} 48$ \\
\hline & $3.60 \pm 0.09$ & 3C 147 \\
\hline \multirow[t]{3}{*}{5.0} & $3.38 \pm 0.05$ & $3 \mathrm{C} 48$ \\
\hline & $3.50 \pm 0.07$ & 3C 147 \\
\hline & $3.46 \pm 0.05$ & $3 \mathrm{C} 286$ \\
\hline \multirow[t]{2}{*}{5.1} & $3.16 \pm 0.10$ & 3C 48 \\
\hline & $3.52 \pm 0.11$ & 3C 147 \\
\hline 5.2 & $3.34 \pm 0.05$ & 3C 286 \\
\hline 5.5 & $3.34 \pm 0.05$ & 3C 147 \\
\hline 5.6 & $3.20 \pm 0.06$ & 3C 147 \\
\hline 6.0 & $2.96 \pm 0.05$ & $3 \mathrm{C} 286$ \\
\hline 6.1 & $2.99 \pm 0.10$ & 3C 147 \\
\hline 6.2 & $2.91 \pm 0.10$ & 3C 147 \\
\hline
\end{tabular}


TABle 3-Continued

\begin{tabular}{ccc}
\hline \hline$\nu(\mathrm{GHz})$ & Flux $(\mathrm{Jy})$ & Calibrator \\
\hline 6.3 & $2.98 \pm 0.10$ & 3C 147 \\
6.4 & $2.90 \pm 0.10$ & 3C 147 \\
6.6 & $2.76 \pm 0.06$ & 3C 147 \\
6.7 & $2.86 \pm 0.06$ & 3C 147 \\
\hline
\end{tabular}


TABLE 4

Flux Density measurements of NGC 253

\begin{tabular}{|c|c|c|c|c|}
\hline $\begin{array}{c}\nu \\
(\mathrm{GHz})\end{array}$ & Core & $\begin{array}{l}\text { Flux (Jy) } \\
\text { Extended }\end{array}$ & Total & Calibrator \\
\hline 1.1 & $2.98 \pm 0.05$ & $3.92 \pm 0.07$ & $6.90 \pm 0.08$ & 3C 147 \\
\hline 1.2 & $2.93 \pm 0.05$ & $3.83 \pm 0.09$ & $6.75 \pm 0.10$ & 3C 147 \\
\hline 1.3 & $2.78 \pm 0.04$ & $3.51 \pm 0.06$ & $6.29 \pm 0.07$ & 3C 147 \\
\hline 1.4 & $2.76 \pm 0.09$ & $3.17 \pm 0.10$ & $5.93 \pm 0.13$ & $3 \mathrm{C} 48$ \\
\hline & $2.75 \pm 0.06$ & $3.22 \pm 0.07$ & $5.97 \pm 0.09$ & 3C 147 \\
\hline 1.5 & $2.54 \pm 0.08$ & $2.59 \pm 0.09$ & $5.13 \pm 0.13$ & $3 \mathrm{C} 48$ \\
\hline 1.7 & $2.48 \pm 0.05$ & $2.68 \pm 0.07$ & $5.16 \pm 0.09$ & 3C 147 \\
\hline 1.8 & $2.39 \pm 0.05$ & $2.50 \pm 0.07$ & $4.89 \pm 0.08$ & 3C 147 \\
\hline 1.9 & $2.36 \pm 0.05$ & $2.42 \pm 0.07$ & $4.78 \pm 0.08$ & 3C 147 \\
\hline 2.0 & $2.35 \pm 0.08$ & $2.29 \pm 0.10$ & $4.64 \pm 0.12$ & $3 \mathrm{C} 48$ \\
\hline 2.1 & $2.25 \pm 0.08$ & $1.96 \pm 0.09$ & $4.21 \pm 0.12$ & $3 \mathrm{C} 48$ \\
\hline 2.6 & $1.95 \pm 0.03$ & $1.80 \pm 0.05$ & $3.75 \pm 0.06$ & 3C 147 \\
\hline 2.7 & $2.01 \pm 0.02$ & $1.74 \pm 0.03$ & $3.75 \pm 0.04$ & $3 \mathrm{C} 147$ \\
\hline 3.0 & $1.98 \pm 0.07$ & $1.76 \pm 0.09$ & $3.74 \pm 0.12$ & $3 \mathrm{C} 48$ \\
\hline 3.1 & $1.89 \pm 0.06$ & $1.70 \pm 0.08$ & $3.59 \pm 0.11$ & $3 \mathrm{C} 48$ \\
\hline & $1.83 \pm 0.02$ & $1.51 \pm 0.03$ & $3.34 \pm 0.04$ & 3C 147 \\
\hline 3.3 & $1.76 \pm 0.02$ & $1.40 \pm 0.04$ & $3.16 \pm 0.04$ & 3C 147 \\
\hline 3.6 & $1.75 \pm 0.04$ & $1.38 \pm 0.07$ & $3.13 \pm 0.08$ & 3C 147 \\
\hline 3.7 & $1.75 \pm 0.08$ & $1.15 \pm 0.17$ & $2.90 \pm 0.19$ & 3C 147 \\
\hline 4.5 & $1.50 \pm 0.02$ & $0.97 \pm 0.04$ & $2.47 \pm 0.04$ & 3C 147 \\
\hline 4.6 & $1.46 \pm 0.04$ & $1.06 \pm 0.07$ & $2.51 \pm 0.08$ & 3C 147 \\
\hline 4.7 & $1.47 \pm 0.03$ & $1.00 \pm 0.09$ & $2.47 \pm 0.10$ & 3C 147 \\
\hline 4.9 & $1.35 \pm 0.04$ & $1.01 \pm 0.07$ & $2.35 \pm 0.08$ & 3C 147 \\
\hline 5.0 & $1.37 \pm 0.10$ & $0.65 \pm 0.15$ & $2.01 \pm 0.18$ & $3 \mathrm{C} 48$ \\
\hline & $1.45 \pm 0.04$ & $0.81 \pm 0.09$ & $2.26 \pm 0.10$ & 3C 147 \\
\hline 5.1 & $1.34 \pm 0.07$ & $0.90 \pm 0.15$ & $2.23 \pm 0.17$ & $3 \mathrm{C} 48$ \\
\hline 5.6 & $1.30 \pm 0.03$ & $0.90 \pm 0.09$ & $2.20 \pm 0.10$ & 3C 147 \\
\hline 5.7 & $1.26 \pm 0.03$ & $0.75 \pm 0.09$ & $2.01 \pm 0.09$ & 3C 147 \\
\hline 5.8 & $1.26 \pm 0.04$ & $0.61 \pm 0.07$ & $1.87 \pm 0.08$ & 3C 147 \\
\hline 5.9 & $1.21 \pm 0.04$ & $0.76 \pm 0.08$ & $1.97 \pm 0.09$ & 3C 147 \\
\hline 6.0 & $1.20 \pm 0.03$ & $0.75 \pm 0.07$ & $1.95 \pm 0.08$ & 3C 147 \\
\hline 6.1 & $1.16 \pm 0.03$ & $0.60 \pm 0.07$ & $1.76 \pm 0.07$ & 3C 147 \\
\hline 6.3 & $1.16 \pm 0.03$ & $0.52 \pm 0.07$ & $1.68 \pm 0.08$ & 3C 147 \\
\hline 6.4 & $1.18 \pm 0.04$ & $0.54 \pm 0.09$ & $1.72 \pm 0.09$ & 3C 147 \\
\hline 6.7 & $1.13 \pm 0.04$ & $0.73 \pm 0.15$ & $1.87 \pm 0.15$ & $3 \mathrm{C} 147$ \\
\hline 7.0 & $1.04 \pm 0.04$ & $0.51 \pm 0.07$ & $1.55 \pm 0.09$ & 3C 147 \\
\hline
\end{tabular}


TABLE 5

Flux DENSITY MEASUREMENTS OF ARP 220

\begin{tabular}{|c|c|c|}
\hline$\nu(\mathrm{GHz})$ & Flux (Jy) & Calibrator \\
\hline \multirow[t]{2}{*}{1.1} & $0.36 \pm 0.02$ & $3 \mathrm{C} 48$ \\
\hline & $0.34 \pm 0.01$ & $3 \mathrm{C} 286$ \\
\hline \multirow[t]{2}{*}{1.2} & $0.37 \pm 0.02$ & $3 \mathrm{C} 48$ \\
\hline & $0.33 \pm 0.01$ & $3 \mathrm{C} 286$ \\
\hline 1.4 & $0.32 \pm 0.01$ & 3C 286 \\
\hline 1.5 & $0.26 \pm 0.02$ & 3C 286 \\
\hline 1.6 & $0.31 \pm 0.03$ & 3C 286 \\
\hline 1.7 & $0.30 \pm 0.01$ & $3 \mathrm{C} 286$ \\
\hline 1.8 & $0.31 \pm 0.01$ & $3 \mathrm{C} 286$ \\
\hline 1.9 & $0.30 \pm 0.01$ & $3 \mathrm{C} 286$ \\
\hline 2.0 & $0.30 \pm 0.01$ & 3C 286 \\
\hline \multirow[t]{2}{*}{2.5} & $0.27 \pm 0.02$ & $3 \mathrm{C} 48$ \\
\hline & $0.29 \pm 0.01$ & 3C 286 \\
\hline \multirow[t]{2}{*}{2.6} & $0.28 \pm 0.02$ & $3 \mathrm{C} 48$ \\
\hline & $0.28 \pm 0.01$ & $3 \mathrm{C} 286$ \\
\hline 2.9 & $0.27 \pm 0.01$ & 3C 286 \\
\hline 3.2 & $0.27 \pm 0.01$ & 3C 286 \\
\hline 3.3 & $0.27 \pm 0.01$ & $3 \mathrm{C} 286$ \\
\hline 3.4 & $0.28 \pm 0.01$ & $3 \mathrm{C} 286$ \\
\hline 3.5 & $0.27 \pm 0.01$ & 3C 286 \\
\hline 3.6 & $0.24 \pm 0.01$ & 3C 286 \\
\hline 4.3 & $0.25 \pm 0.02$ & 3C 286 \\
\hline 4.4 & $0.23 \pm 0.02$ & 3C 286 \\
\hline 4.5 & $0.24 \pm 0.02$ & $3 \mathrm{C} 286$ \\
\hline 4.6 & $0.24 \pm 0.02$ & $3 \mathrm{C} 286$ \\
\hline 4.7 & $0.26 \pm 0.02$ & $3 \mathrm{C} 286$ \\
\hline \multirow{2}{*}{5.0} & $0.24 \pm 0.02$ & $3 \mathrm{C} 48$ \\
\hline & $0.23 \pm 0.01$ & 3C 286 \\
\hline 5.2 & $0.23 \pm 0.01$ & $3 \mathrm{C} 286$ \\
\hline 5.4 & $0.24 \pm 0.01$ & $3 \mathrm{C} 286$ \\
\hline \multirow[t]{2}{*}{5.7} & $0.20 \pm 0.02$ & $3 \mathrm{C} 48$ \\
\hline & $0.22 \pm 0.01$ & 3C 286 \\
\hline 6.0 & $0.22 \pm 0.01$ & $3 \mathrm{C} 286$ \\
\hline 6.2 & $0.29 \pm 0.03$ & $3 \mathrm{C} 286$ \\
\hline \multirow[t]{2}{*}{6.3} & $0.20 \pm 0.03$ & $3 \mathrm{C} 48$ \\
\hline & $0.20 \pm 0.01$ & $3 \mathrm{C} 286$ \\
\hline 6.7 & $0.23 \pm 0.03$ & $3 \mathrm{C} 286$ \\
\hline 7.0 & $0.19 \pm 0.02$ & $3 \mathrm{C} 286$ \\
\hline
\end{tabular}


TABle 6

High-Frequency observations. See 4.1 for discussion.

\begin{tabular}{|c|c|c|c|c|c|}
\hline $\begin{array}{c}\nu \\
(\mathrm{GHz})\end{array}$ & $(\mathrm{Jy})^{\text {Flux }}$ & Telescope & Tel. Kind ${ }^{\mathrm{a}}$ & $\begin{array}{c}\theta_{\mathrm{LAS}}{ }^{\mathrm{b}} \\
(\operatorname{arcsec})\end{array}$ & Reference \\
\hline \multicolumn{6}{|c|}{ M82 } \\
\hline 22.4 & $1.00 \pm 0.15$ & VLA D-cfg. & I & 45 & Carlstrom \& Kronberg (1991) \\
\hline 23 & $1.36 \pm 0.07$ & Effelsberg $100 \mathrm{~m}$ & $\mathrm{SD}$ & & Kronberg et al. (1979) \\
\hline 24.5 & $1.19 \pm 0.03$ & Effelsberg $100 \mathrm{~m}$ & $\mathrm{SD}$ & & Klein et al. (1988) \\
\hline 32 & $1.02 \pm 0.06$ & Effelsberg $100 \mathrm{~m}$ & $\mathrm{SD}$ & & Klein et al. (1988) \\
\hline 42.2 & $0.82 \pm 0.16$ & VLA C-cfg. & I & 43 & Rodríguez-Rico et al. (2004) \\
\hline 87.2 & $0.51 \pm 0.08^{\mathrm{c}}$ & Kitt Peak 11m & SD & & Jura et al. (1978) \\
\hline 92 & $0.59 \pm 0.09$ & BIMA & I & 50 & Carlstrom \& Kronberg (1991) \\
\hline 92 & $0.67 \pm 0.10$ & OVRO mm Arr. & I & 55 & Seaquist et al. (1996) \\
\hline \multicolumn{6}{|c|}{ NGC 253 Core Component } \\
\hline 22 & $0.49 \pm 0.05$ & ATCA EW367-cfg. & $\mathrm{I}$ & 61 & Ricci et al. $(2006)$ \\
\hline 23.7 & $0.56 \pm 0.06$ & VLA DnC-cfg. & I & 60 & Takano et al. (2005) \\
\hline 24 & $0.52 \pm 0.05$ & ATCA EW367-cfg. & I & 56 & Ott et al. $(2005)$ \\
\hline 94 & $0.32 \pm 0.03$ & BIMA A/B/C-cfg. & I & 103 & Peng et al. (1996) \\
\hline \multicolumn{6}{|c|}{ Arp 220} \\
\hline 22.5 & $0.090 \pm 0.006$ & VLA A-cfg. & $\mathrm{I}$ & 2 & Zhao et al. (1996) \\
\hline 23.1 & $0.034 \pm 0.007^{\mathrm{d}}$ & VLA A-cfg. & I & 2 & Norris $(1988)$ \\
\hline 42.2 & $0.044 \pm 0.004$ & VLA C-cfg. & I & 43 & Rodríquez-Rico et al. (2005) \\
\hline 87 & $0.030 \pm 0.006^{\mathrm{d}}$ & RAINBOW AB-cfg. & I & 8 & Imanishi et al. (2007) \\
\hline 87 & $0.035 \pm 0.007^{\mathrm{d}}$ & IRAM PdBI & $\mathrm{I}$ & 40 & Radford et al. (1991) \\
\hline 97.2 & $0.061 \pm 0.010$ & IRAM 30m & $\mathrm{SD}$ & & Anantharamaiah et al. (2000) \\
\hline
\end{tabular}

aThe letter "I" denotes an interferometer, "SD" denotes a single dish.

${ }^{\mathrm{b}}$ Largest angular scale to which the observation is sensitive.

${ }^{\mathrm{c}}$ Converted to flux density scale of Baars et al. (1977).

${ }^{\mathrm{d}}$ Uncertainty of $20 \%$ adopted. 
TABLE 7

RESUltS OF SPECTRAL MODELING.

\begin{tabular}{cccc}
\hline \hline Parameter $^{\mathrm{a}}$ & M82 & NGC 253 & Arp 220 \\
\hline$\sqrt{\chi_{r}^{2}}$ & 1.21 & 1.02 & 1.17 \\
$A$ & $0.93 \pm 0.01$ & $0.50 \pm 0.01$ & $-0.48_{-0.011}^{+0.007}$ \\
$B$ & $-0.56 \pm 0.02$ & $-0.51 \pm 0.03$ & $-0.01_{-0.04}^{+0.03}$ \\
$C$ & $-0.12 \pm 0.03$ & $-0.16 \pm 0.03$ & $-0.31_{-0.04}^{+0.02}$ \\
$\tau_{1}$ & $0.04_{-0.01}^{+0.01}$ & $0.27 \pm 0.06$ & $<0.06$ \\
& & & \\
$\alpha_{5}$ & $-0.73 \pm 0.04$ & $-0.74 \pm 0.04$ & $-0.44_{-0.05}^{+0.01}$ \\
$\Delta$ & $-0.24 \pm 0.07$ & $-0.33 \pm 0.06$ & $-0.62_{-0.08}^{+0.04}$ \\
$f_{\text {th }}$ & $0.06 \pm 0.02$ & $0.07_{-0.01}^{+0.03}$ & $<0.02$ \\
\hline
\end{tabular}

${ }^{a}$ Uncertainties in the parameters are marginalized $1 \sigma$ values determined via Monte Carlo simulations with 10000 realizations for each source. 


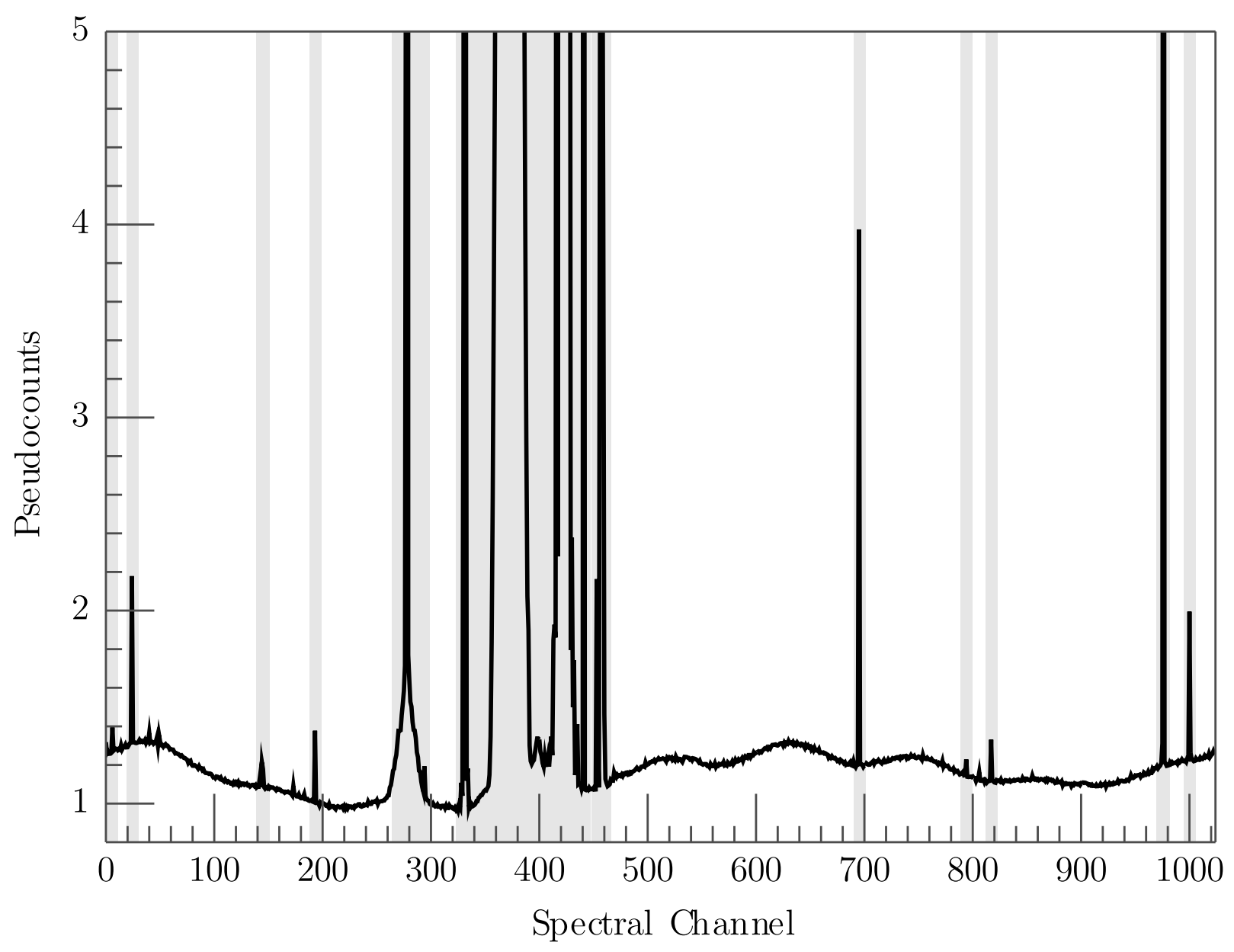

Fig. 1.- An assessment of the RFI present during a block of $1.7 \mathrm{GHz}$ observations. This plot is typical of those produced during execution of the semi-automated RFI excision algorithm described in $\$ 3.1$ Solid lines show summed amplitudes of the $1.7 \mathrm{GHz}$ data obtained during segment \#14 as a function of correlator channel, corresponding to frequencies ranging from 1.65 to $1.75 \mathrm{GHz}$. Shaded regions indicate RFI-afflicted regions detected by the peak-finding algorithm. Plots such as these were reviewed and sometimes edited to improve the flagging (e.g., to widen the flagged region around the broad peak centered near channel 280). Channel 512 corresponds to a sky frequency of $1.700 \mathrm{GHz}$ and the channel width is $102.4 \mathrm{kHz}$. The sinusoidal features across the bandpass are due to the ATA digital filter. 


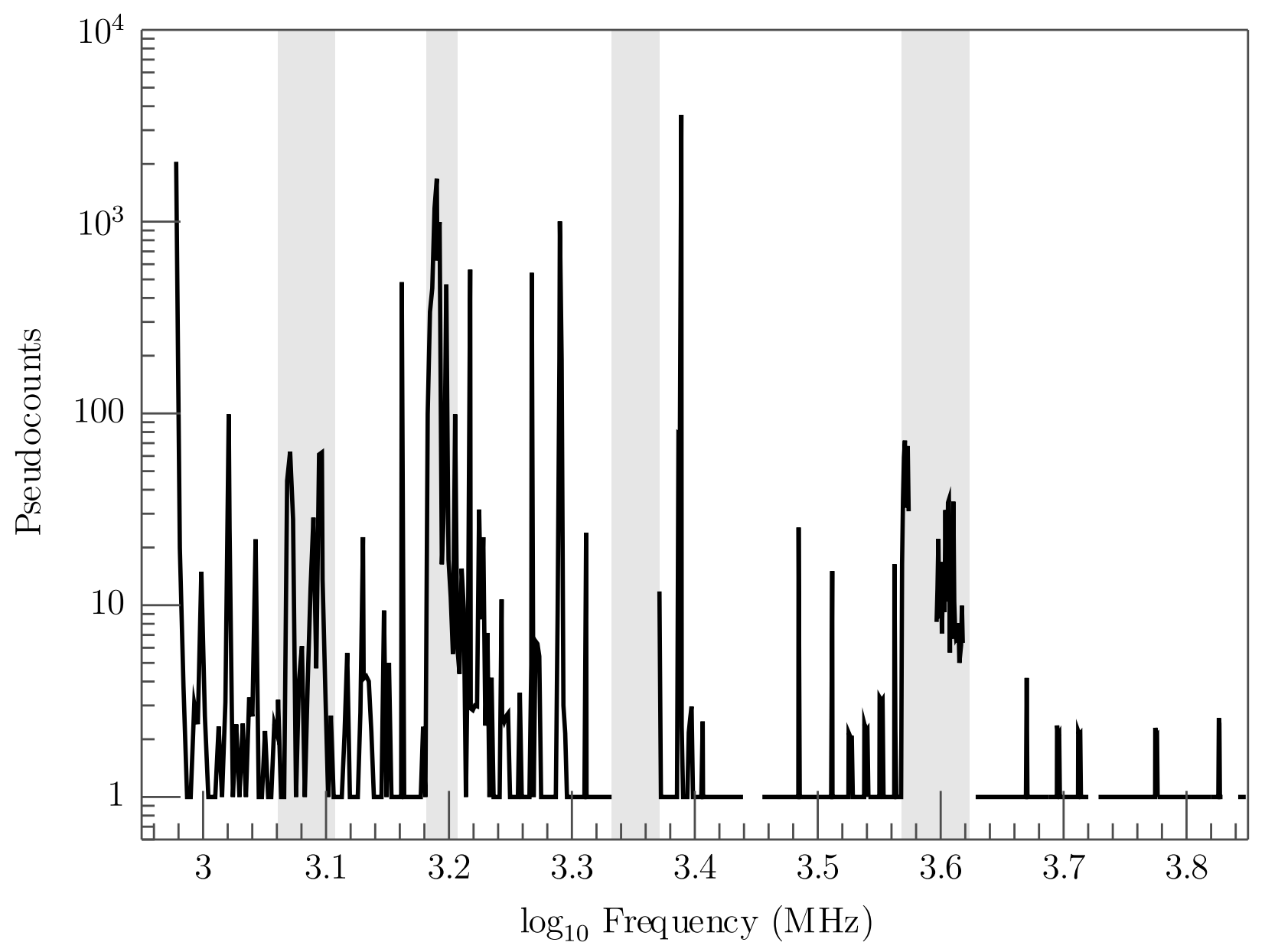

Fig. 2.- The RFI environment of the ATA, in schematic form. Solid lines show a processed version of the summed data amplitudes, as described in 83.1 . The raw $100 \mathrm{kHz}$ channelized amplitudes were processed to increase clarity while accurately portraying narrow RFI peaks. Gaps in the data are due to a lack of observations, usually because the RFI was known to dominate astronomical signals at certain frequencies. The shaded regions indicate bands afflicted with especially strong and persistent RFI. 


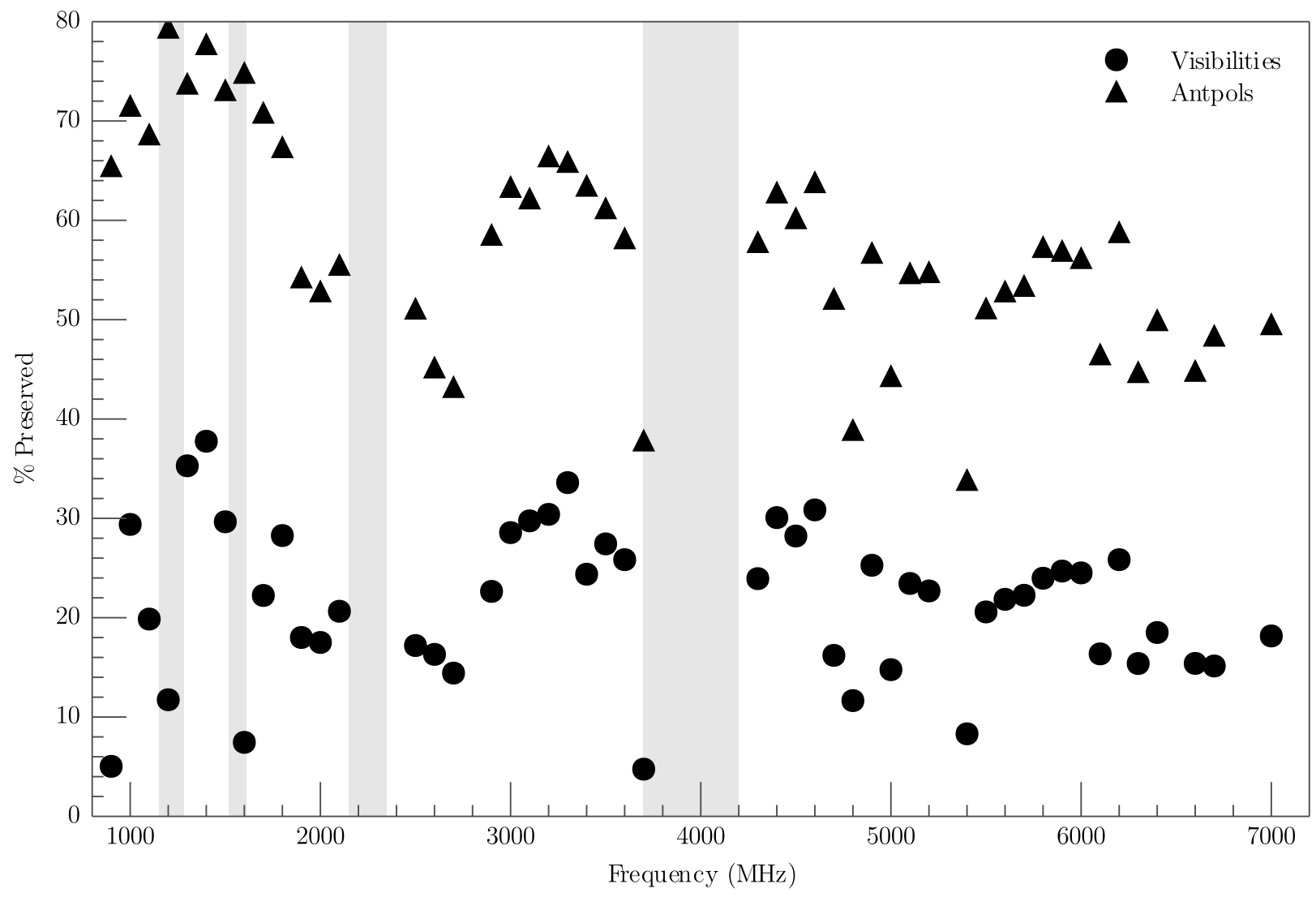

Fig. 3.- Average data retention rates over all observations, as a function of observing frequency. Entirely discarded datasets are not shown. Triangles denote the fraction of antpols used during imaging as compared to the number present in the raw data. Circles denote the same for individual visibilities. The shaded regions represent bands afflicted with strong, persistent RFI. The maximum possible visibility retention rate is $\sim 75 \%$ due to the flagging of edge channels, shadowed antennas, and short baselines described in \$3.1. 

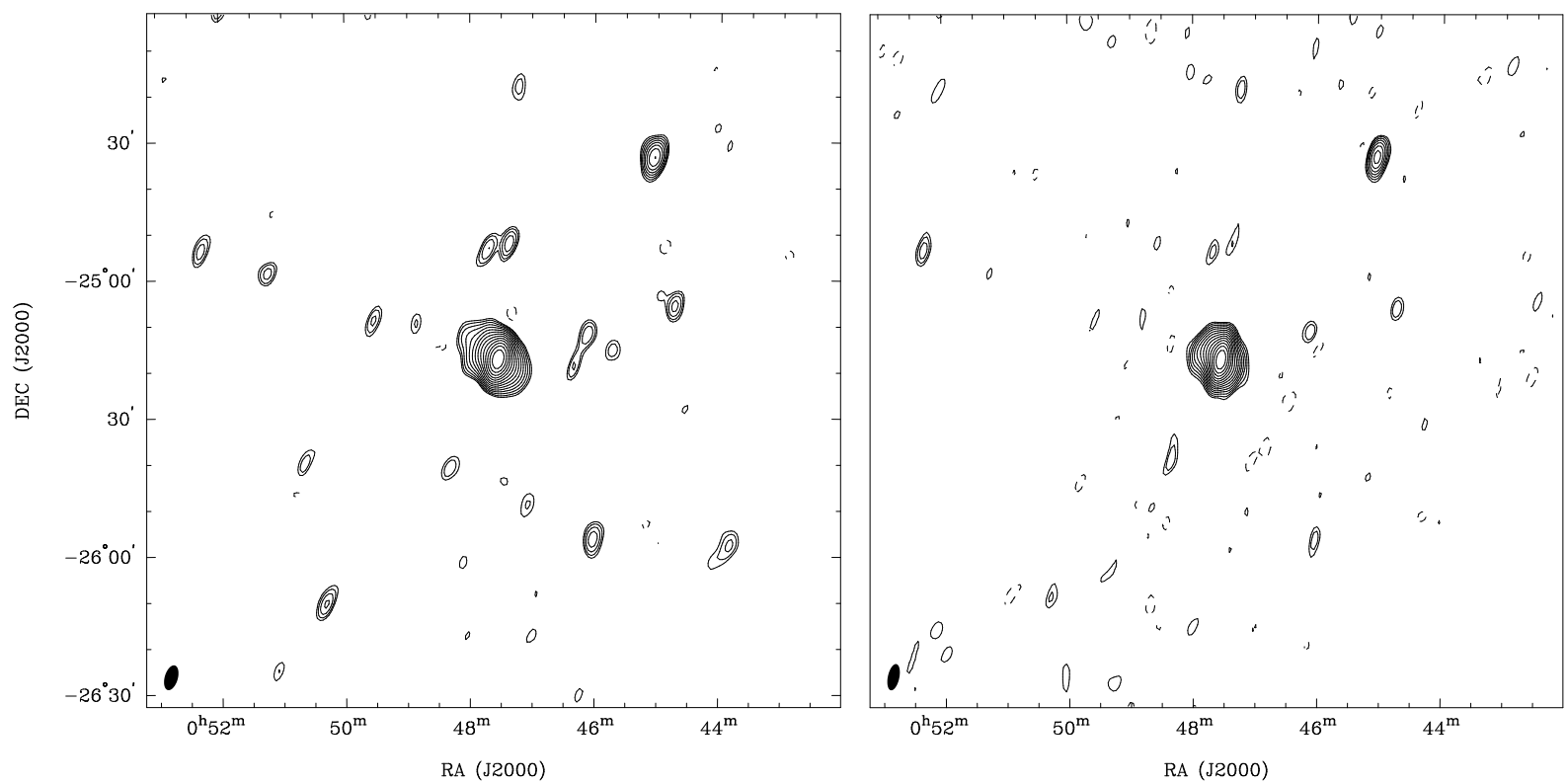

Fig. 4.- ATA maps of NGC 253 at $1.3 \mathrm{GHz}$. The left panel shows the reference image for this source and frequency while the right panel shows an image generated from a 70 s integration made during Segment \#3. The total integration time for the reference image is $250 \mathrm{~s}$. Contour levels in both panels are $30 \mathrm{mJy} \times 2^{n / 2}$ for $n=0,1,2, \ldots, 13$. The RMS noise levels of the reference image and the snapshot are 9 and $11 \mathrm{mJy}$, respectively. Although the longer integration time and more complete $u$ - $v$ coverage of the reference image lead to a noticeable increase in its quality, the total fluxes of NGC 253 measured in the two images agree to $2 \%$. 


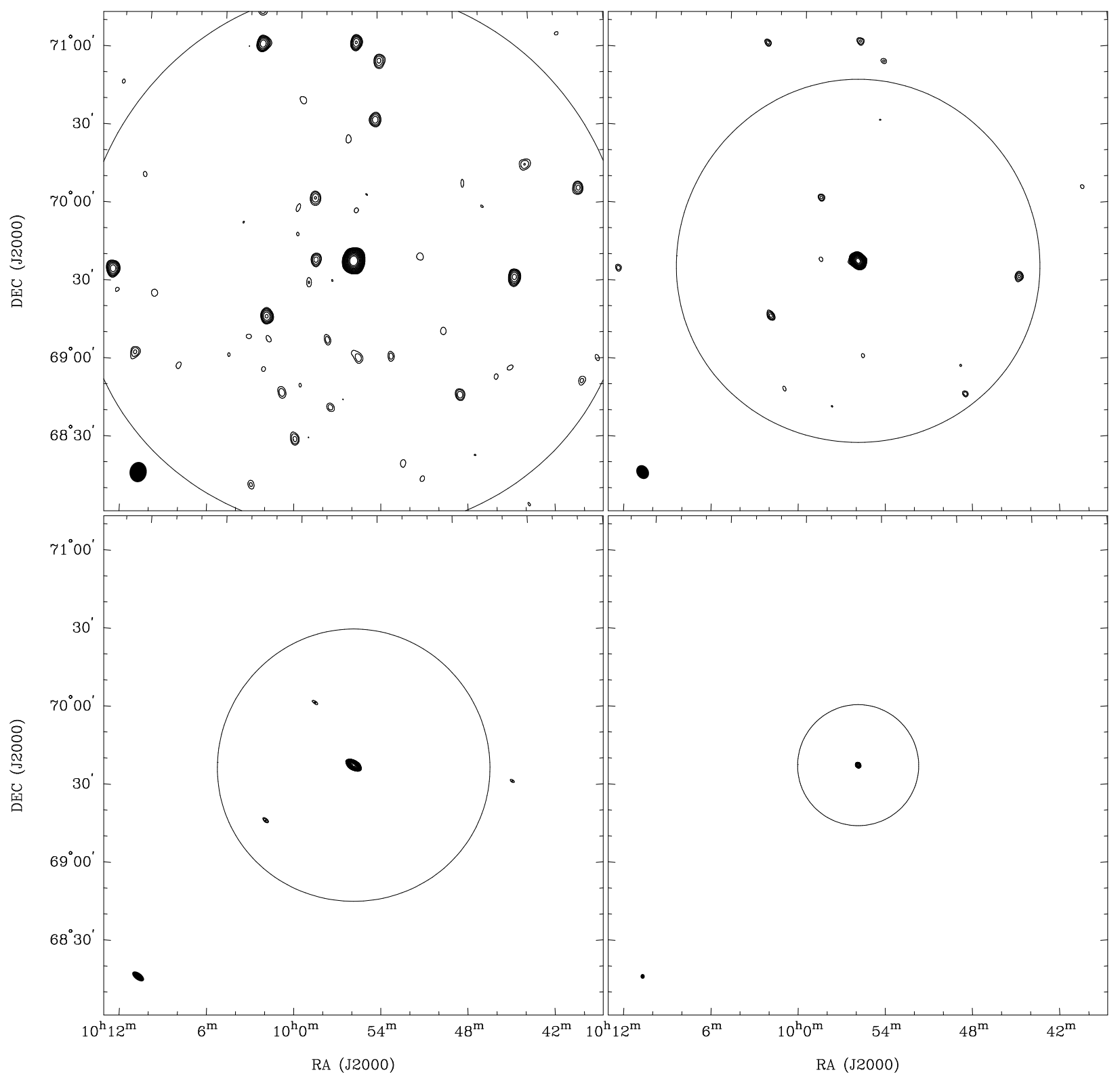

Fig. 5.- ATA maps of M82 at 1.0, 1.5, 2.0, and $4.5 \mathrm{GHz}$ (top left, top right, bottom left, and bottom right panels, respectively). The depicted maps are the reference images at each frequency reimaged onto a common coordinate grid. Circles: half-power points of the ATA beam. Filled ellipses: synthesized beamsizes. Contour levels in all panels are $50 \mathrm{mJy} \times 2^{n / 2}$ for $n=0,1,2, \ldots, 13$. The RMS noise levels of the images are 17, 13, 10, and $11 \mathrm{mJy}$. The number of sources seen decreases as frequency increases due to both the shrinking primary beam of the telescope and the general downward slope of the radio spectra of celestial sources. 


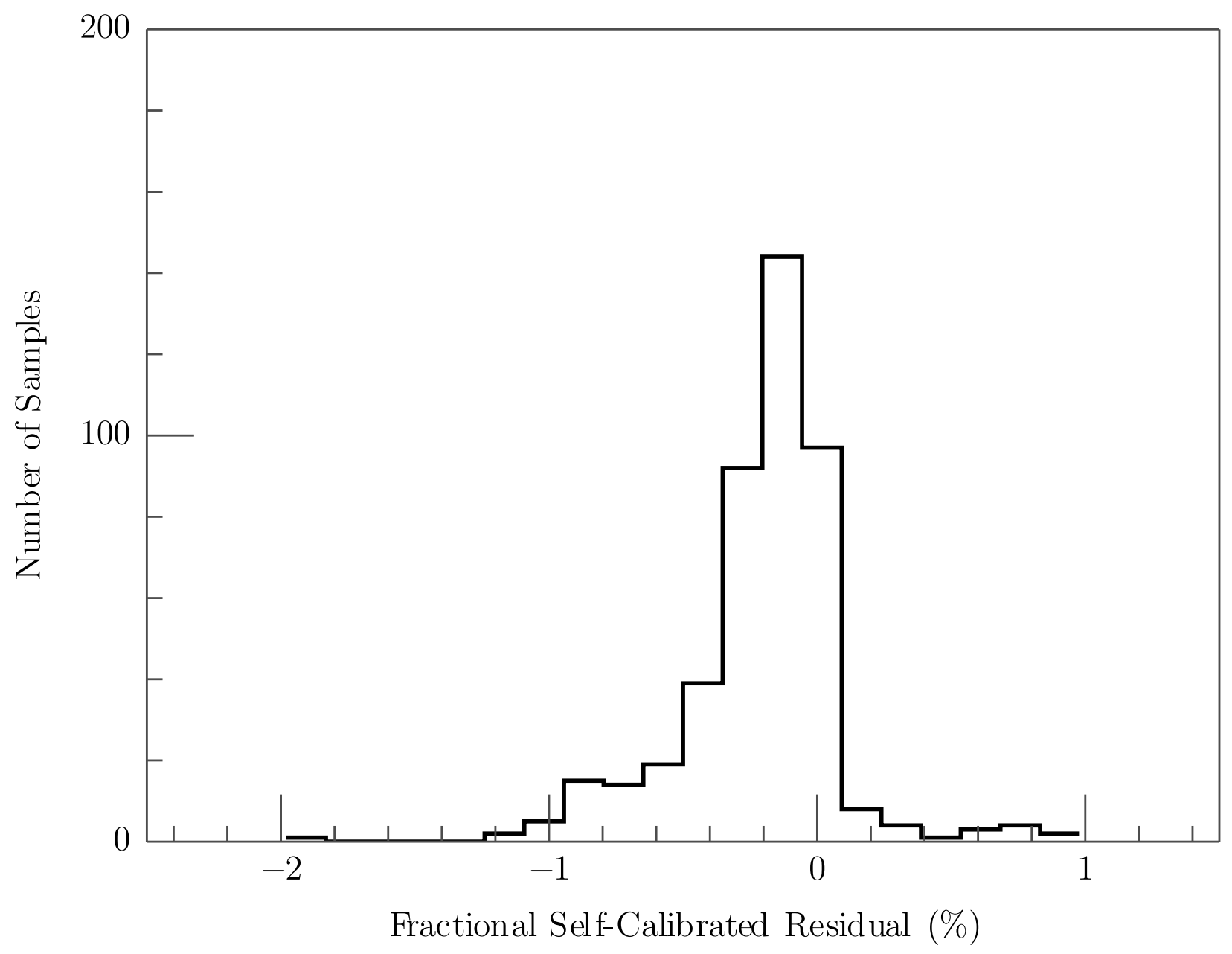

Fig. 6.- Histogram of fractional flux residuals between calibrator observations and analytic calibrator models. The fluxes for the calibrator observations are derived from $u-v$ modeling of calibrator data that have been self-calibrated to the calibrator reference images. 


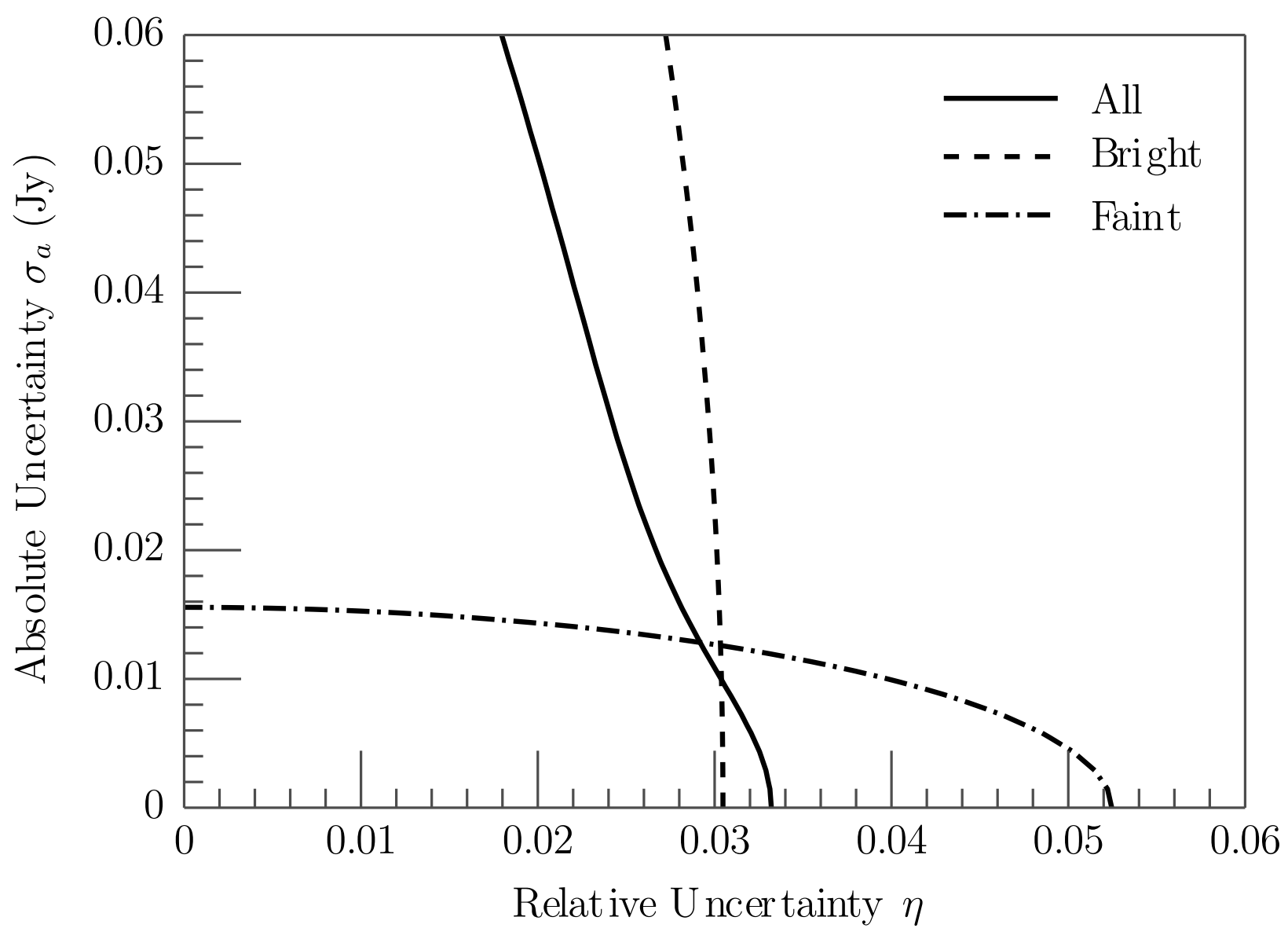

Fig. 7.- Contours of $K^{2}=k$ in the $\eta-\sigma_{a}$ space of uncertainty augmentation parameters. See $\$ 3.4$ for discussion. Solid line: the contour for $K^{2}$ values derived from all repeated flux measurements. Dashed line: the contour for values derived from measurements of sources with $S_{\nu}>3 \mathrm{Jy}$. Dot-dashed line: the contour for values derived from measurements of sources with $S_{\nu}<1 \mathrm{Jy}$. 

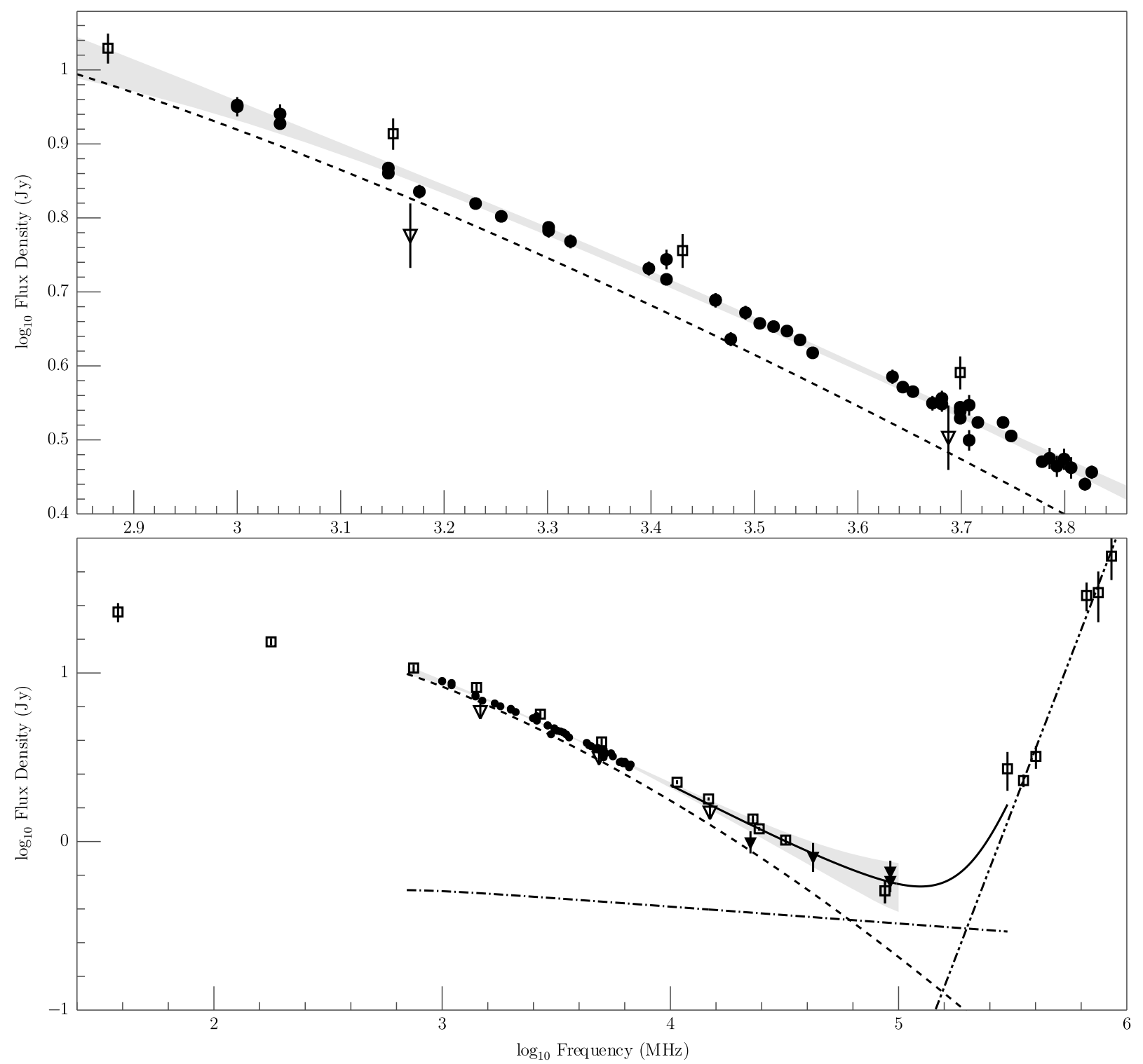

Fig. 8. - Spectrum of M82 with model. The two panels show different views of the same data. Circles: new measurements presented in this paper. Other symbols indicate data from the literature: Carlstrom \& Kronberg (1991) and citations therein; Klein et al. (1988) and citations therein; Jura et al. (1978); Kronberg et al. (1979); Leeuw \& Robson (2009); Rodríguez-Rico et al. (2004); Seaquist et al. (1996). These measurements are made with various telescopes in various configurations and, due to the properties of interferometers, are not always directly comparable. Squares: data obtained with single dishes. Triangles: data obtained with interferometers. Filled symbols: data used in the fits described in 4 4.1.1. Open symbols: data not included in the fits. Shaded region: the $3 \sigma$ uncertainty region of the model spectrum as described in $\$ 4.2$ Dashed line: the model nonthermal contribution. Dash-dotted line: the model thermal contribution. Dash-double-dotted line: a power-law fit to the FIR data. Solid line: the sum of the thermal, nonthermal, and FIR power law contributions. See $\$ 4.2 .1$ for further discussion of the model results. 


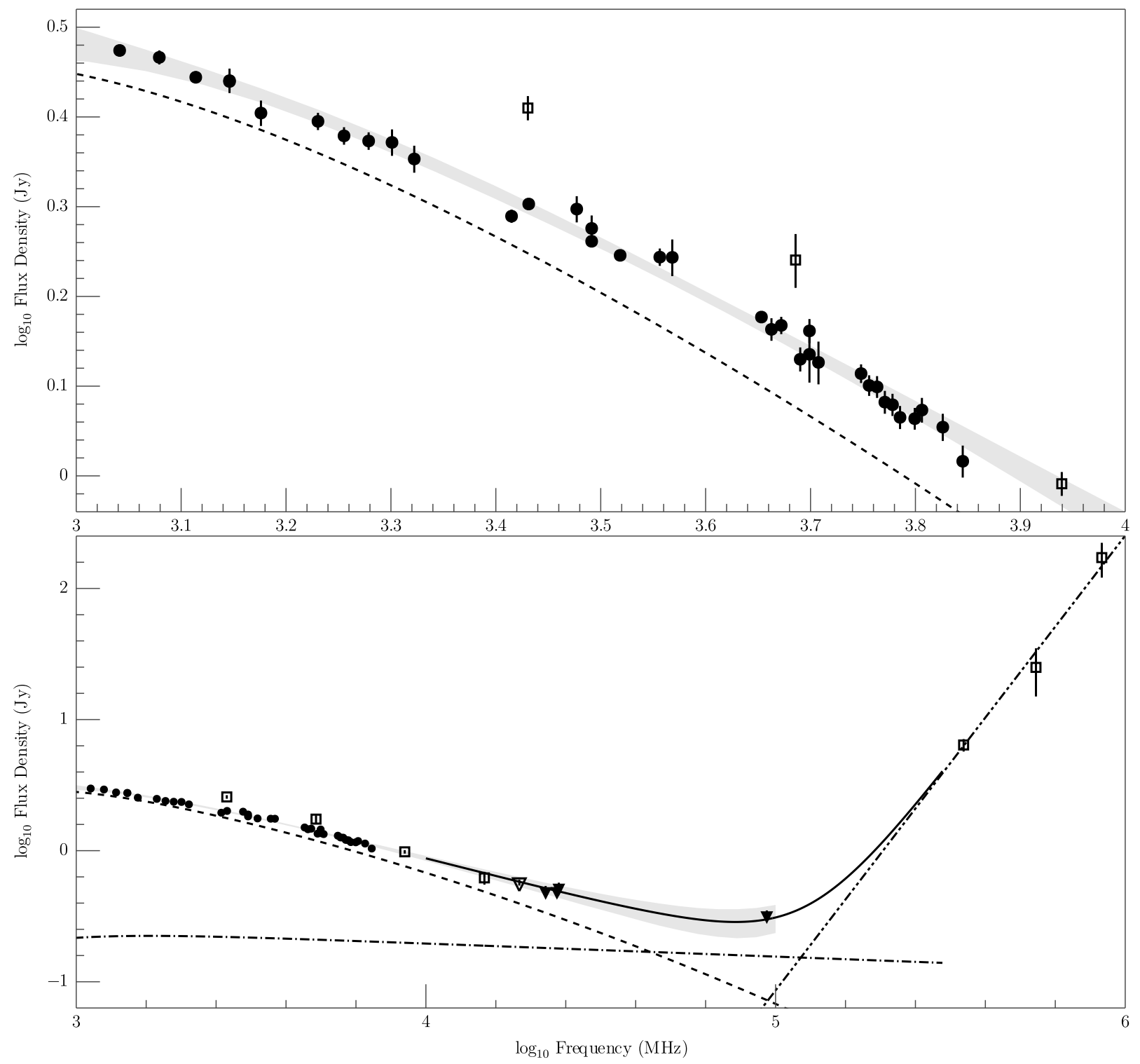

Fig. 9.- Spectrum of the core component of NGC 253 with model. The symbols are as in Figure 8 External data are found in Beck et al. (1979); Hildebrand et al. (1977); Ott et al. (2005); Peng et al. (1996); Ricci et al. (2006); Rieke et al. (1973); Takano et al. (2005); Weiß et al. (2008). See $\$ 4.2 .2$ for discussion. 


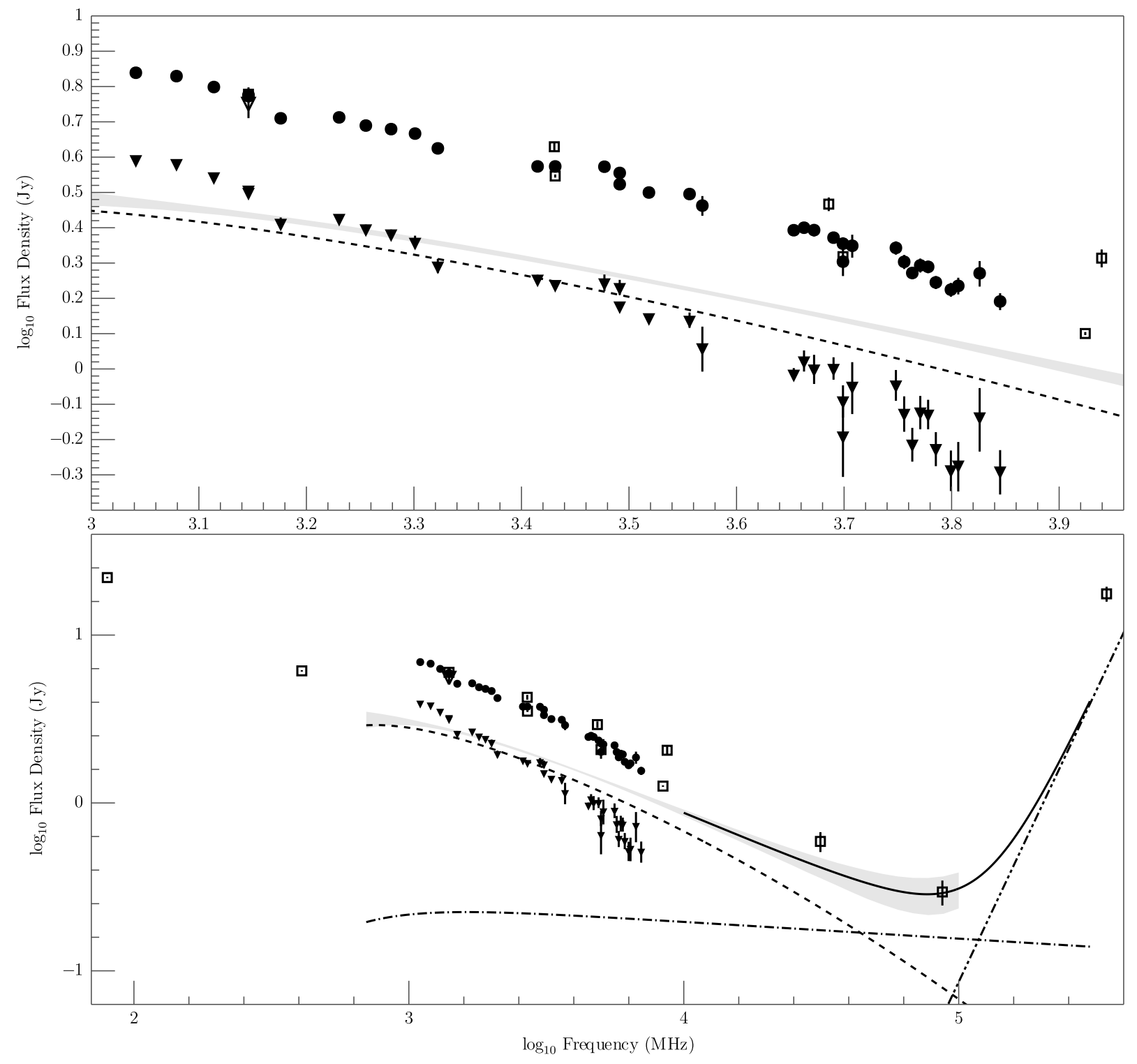

Fig. 10. - Spectra of the extended component and total flux of NGC 253. The model used in Figure 9 is overlaid to ease comparison. External data are found in Beck et al. (1979); Geldzahler \& Witzel (1981); Jura et al. (1978); Wright \& Otrupcek (1990); Strickland et al. (2004); Weiß et al. (2008). Triangles: fluxes of the extended component of NGC 253. Circles: sum of extended and core component fluxes. Other symbols are as in the previous plot. 

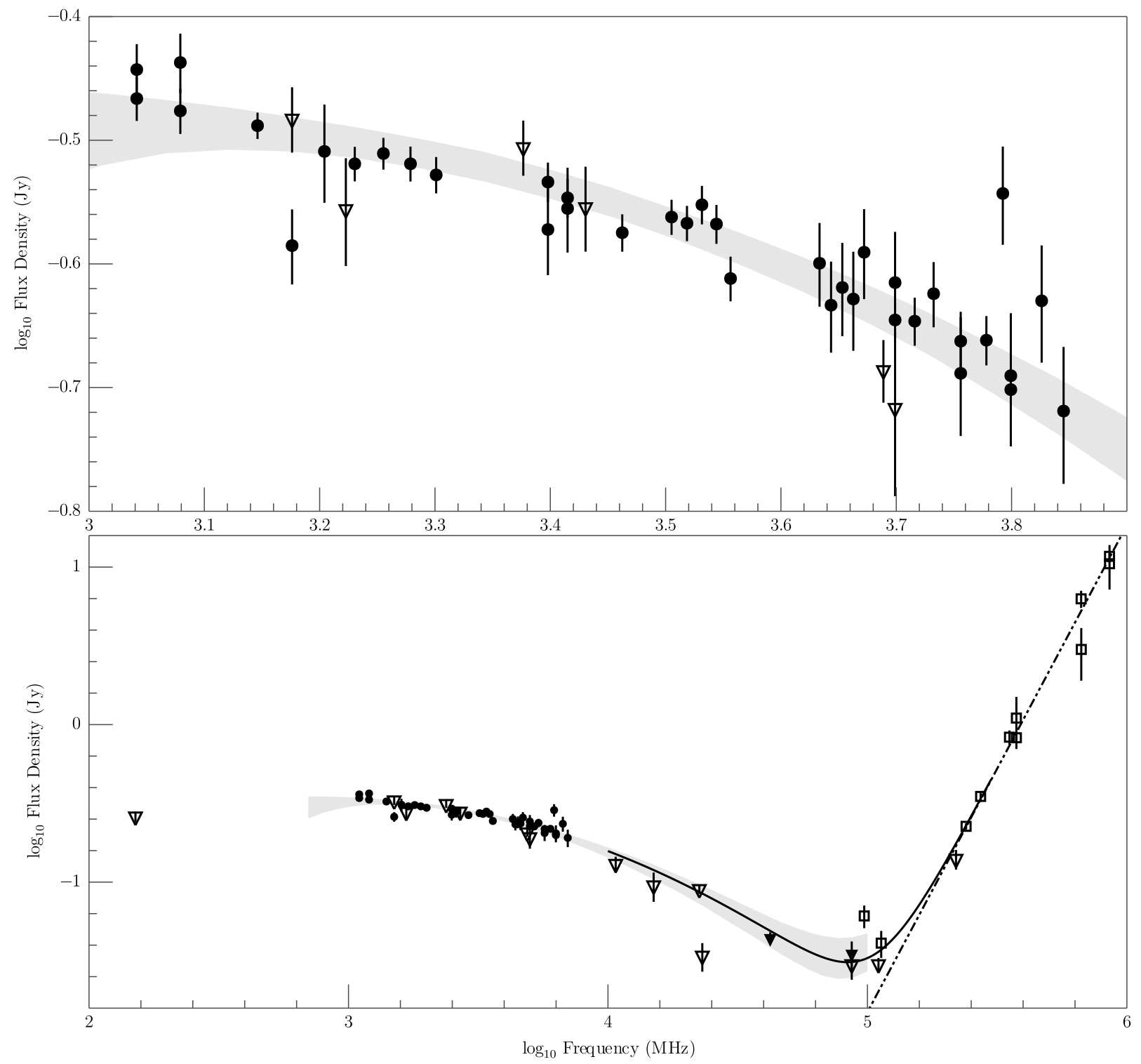

Fig. 11.- Spectrum of Arp 220 with model. The symbols are as in Figure 8, See 4.2 .3 for further discussion. External data are found in Anantharamaiah et al. (2000); Carico et al. (1992); Dunne \& Eales (2001); Eales et al. (1989); Imanishi et al. (2007); Norris (1988); Radford et al. (1991); Rigopoulou et al. (1996); Rodríguez-Rico et al. (2005); Scoville et al. (1991); Sopp \& Alexander (1991); Woody et al. (1989); Zhao et al. (1996) . 


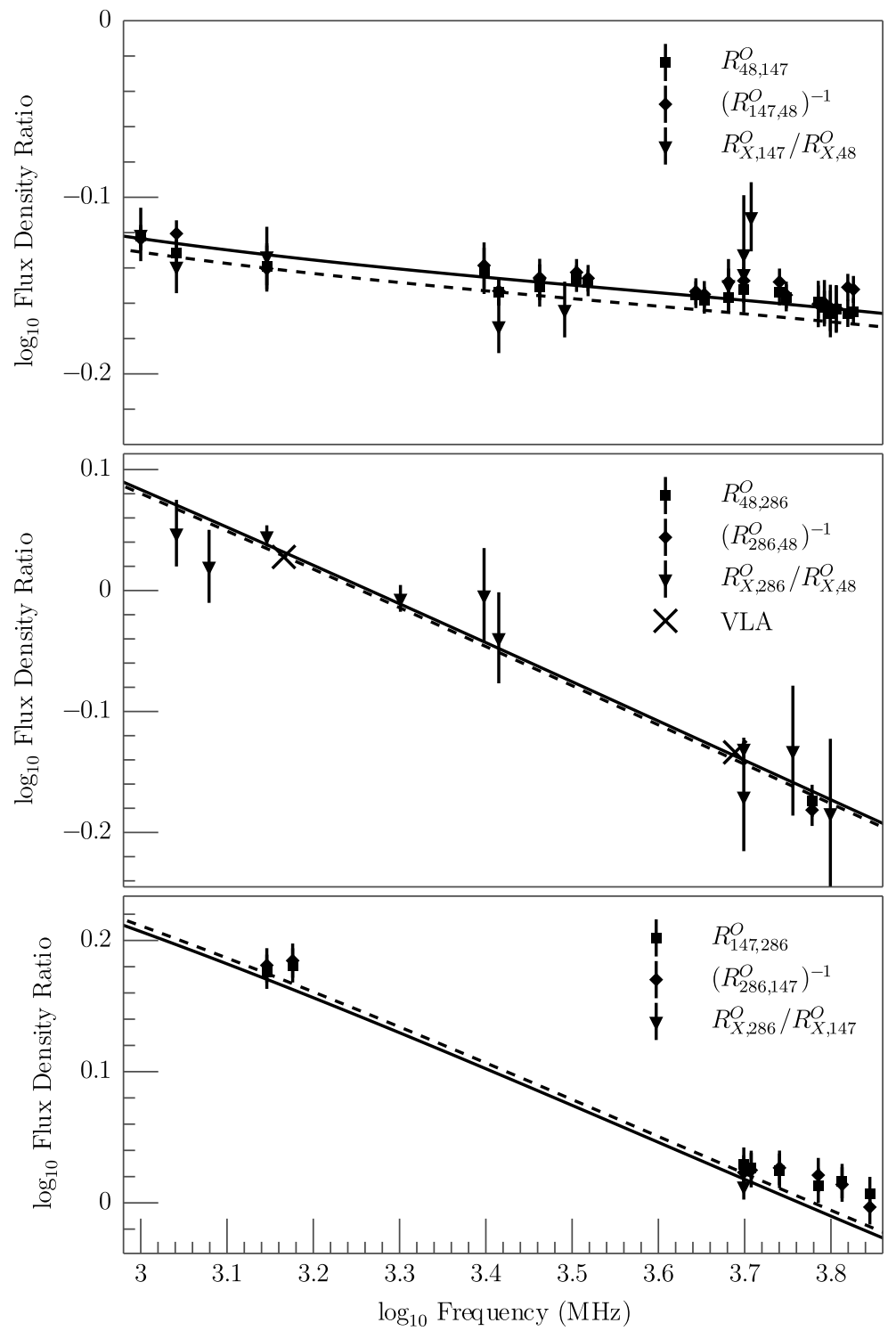

Fig. 12.- Flux density ratios of calibrator observations and spectral models. See $\$ 3.6$ for definitions. Dashed lines: ratios of the VLA 1999.2 models. Solid lines: ratios of the spectral models adopted in this work. Squares and diamonds: directly observed calibrator ratios. The good agreement between the original and inverted ratios indicates that there is negligible flux loss in the secondary calibration process ( 93.2 .2$)$. Triangles: calibrator ratios computed indirectly from the ratio of two observations of a science target. Xs: measurements of $R_{48,286}^{O}$ made at the VLA in 2008 September. The observations of $R_{147,286}^{O}$ were made in 2009 June and exhibit discrepant behavior; see $\$ 3.6$ for discussion. 


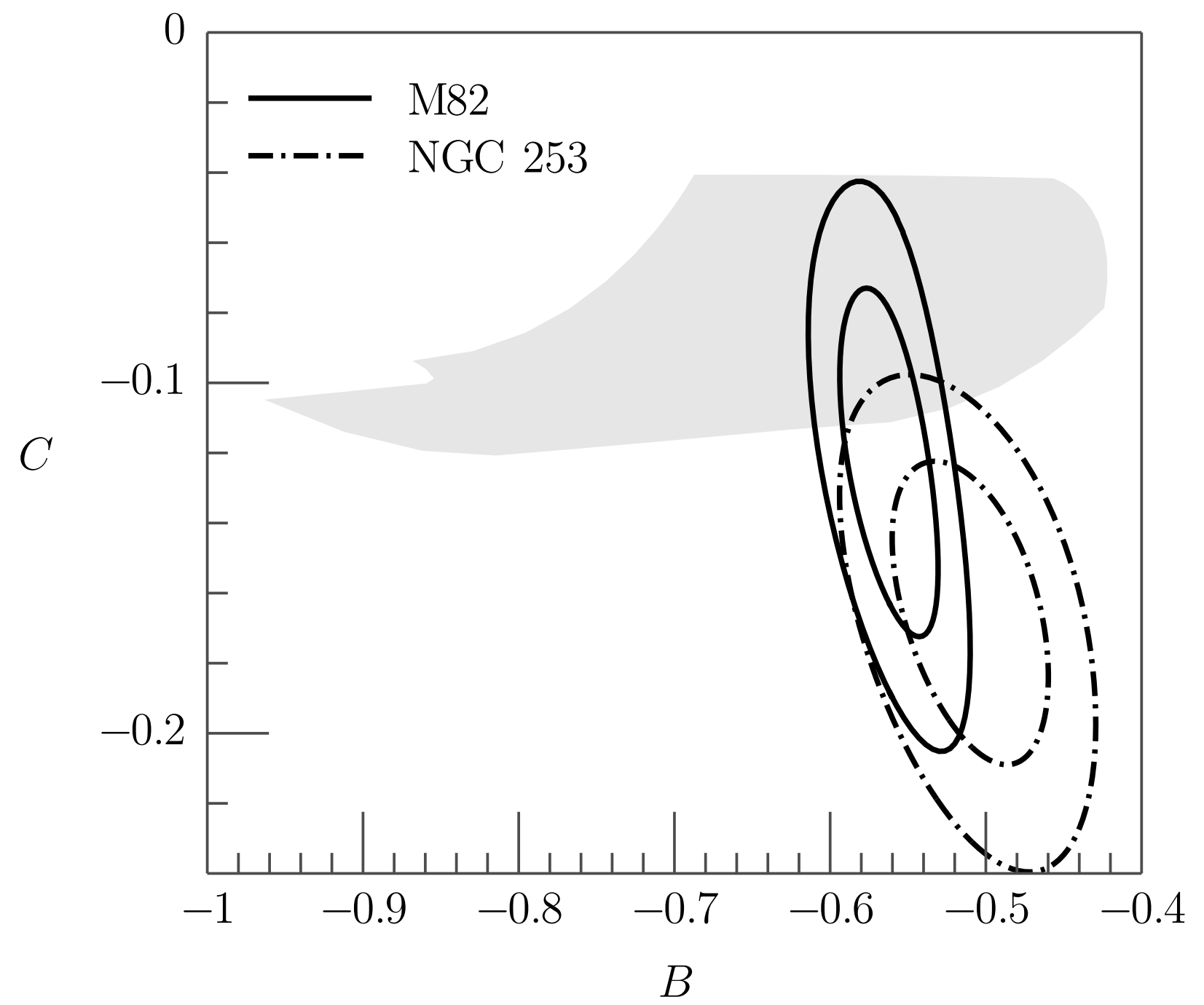

Fig. 13.- Observationally and theoretically derived values of the spectral curvature parameters $B$ and $C$. Contours: $1 \sigma$ (inner) and $2 \sigma$ (outer) confidence regions for the parameters as derived from the modeling described in Shaded region: the range of $B$ and $C$ values taken by the model of $\mathrm{T}+06$ over a range of parameters appropriate for M82 as described in \$5. The equivalent region for the NGC 253 parameters is virtually identical to the one shown. 


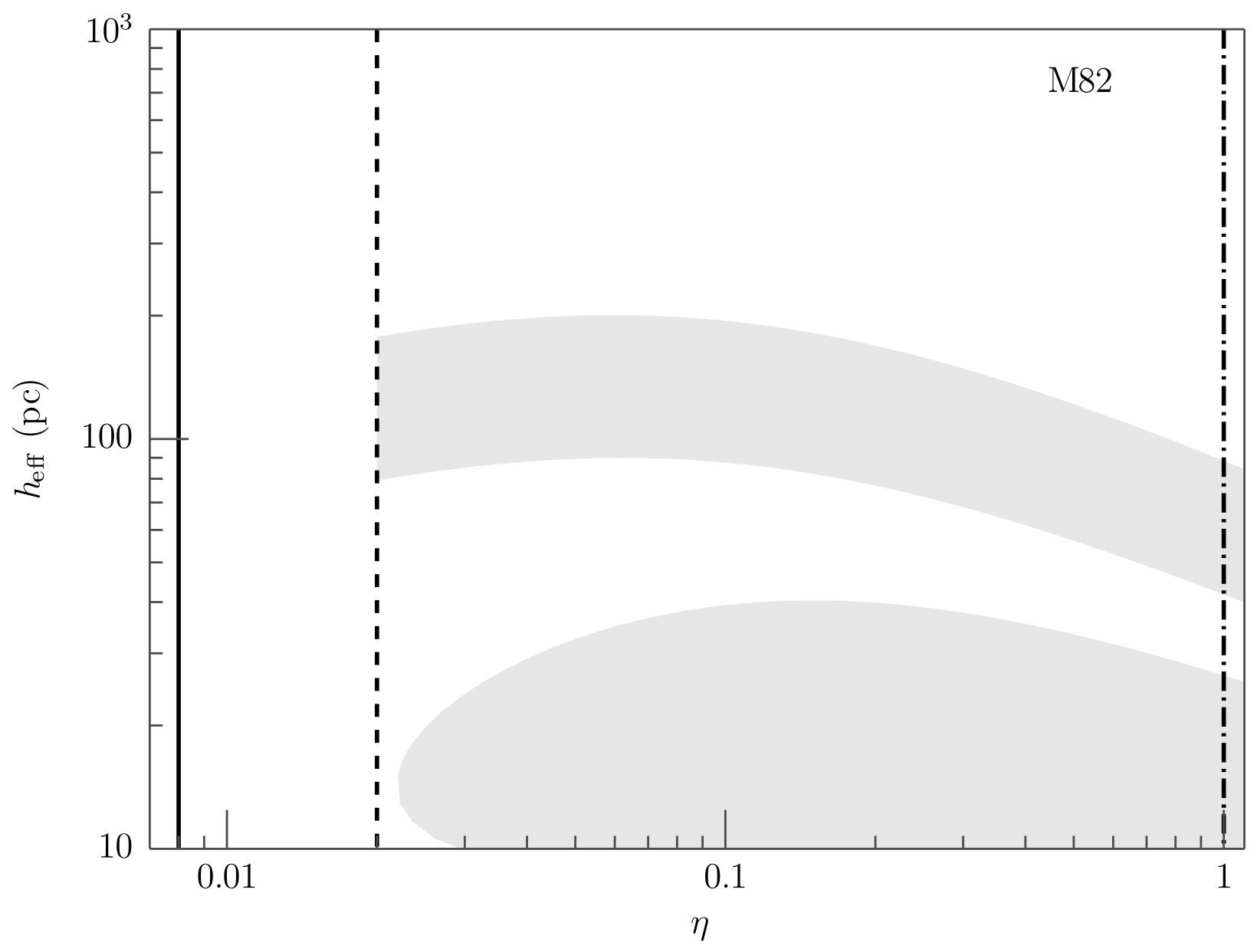

Fig. 14.- Values of $\eta$ and $h_{\text {eff }}$ for which spectral curvature parameters $B$ and $C$ derived from the T+06 model are consistent with observations of M82 to within $2 \sigma$. Upper shaded region: the allowed parameters for $p=2$. Lower shaded region: the allowed parameters for $p=2.3$. Solid line: the value of $\eta$ such that $B=B_{\min }$. Dashed line: the value of $\eta$ such that the synchrotron cooling timescale $\tau_{\text {syn }}$ and the escape time $\tau_{\mathrm{esc}}$ of $\mathrm{T}+06$ are equal, assuming $\nu=1.43 \mathrm{GHz}, h_{\mathrm{eff}}=100 \mathrm{pc}$, and the galactic wind speed $v_{w}=500 \mathrm{~km} \mathrm{~s}^{-1}$. This defines the approximate lower limit in $\eta$ of the validity of the $\mathrm{T}+06$ numerical model. Dot-dashed line: the value of $\eta$ such that $B=B_{\text {eq }}$. 


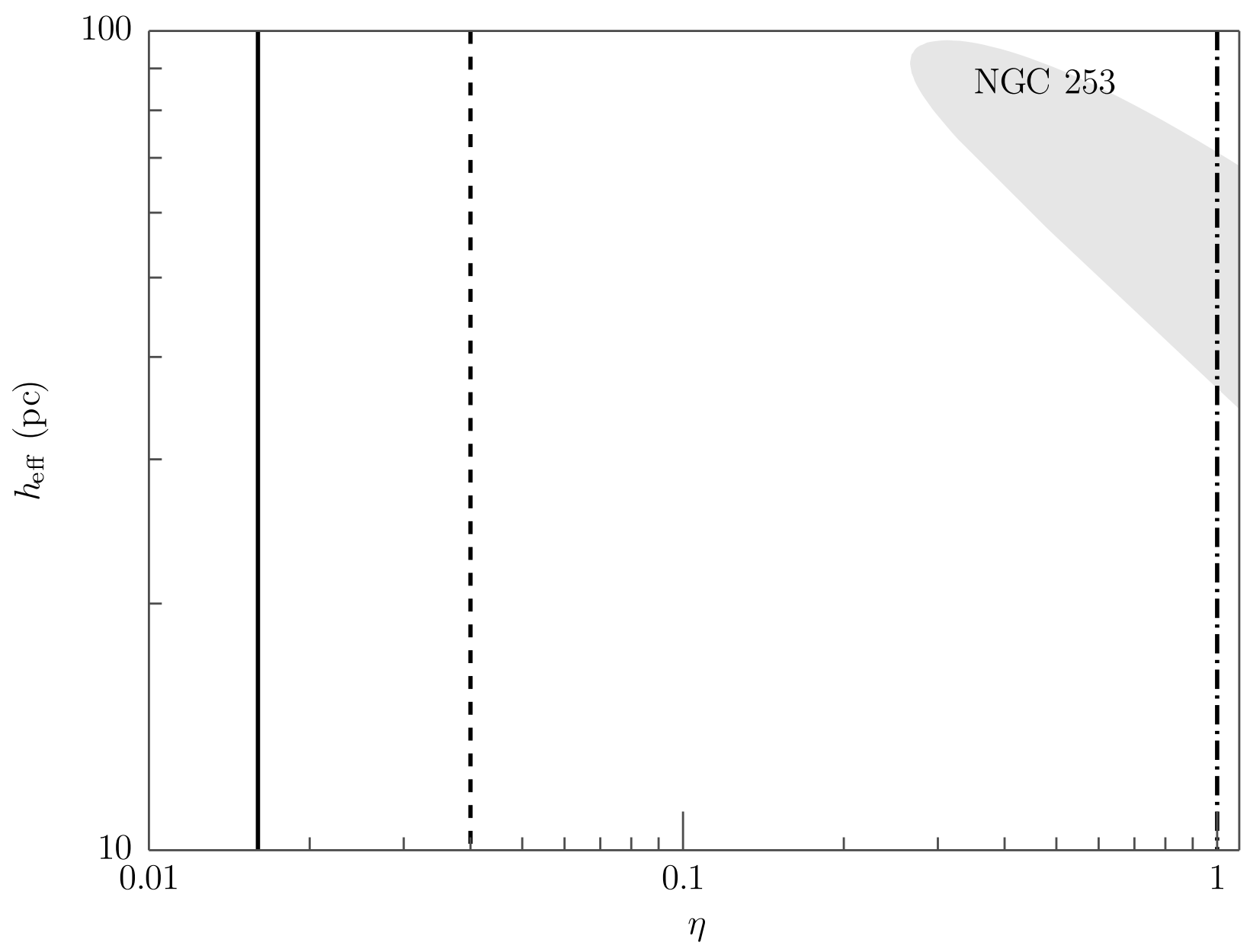

Fig. 15. - Values of $\eta$ and $h_{\text {eff }}$ for which spectral curvature parameters $B$ and $C$ derived from the $\mathrm{T}+06$ model are consistent with observations of NGC 253 to within $2 \sigma$. The symbols are as in the previous figure. Shaded region: the allowed parameters for $p=2$. The $\mathrm{T}+06$ model with $p=2.3$ is excluded to a $99.99 \%$ confidence limit. 